\title{
The intraflagellar transport protein IFT20 controls lysosome biogenesis by regulating the post-Golgi transport of acid hydrolases
}

\author{
Francesca Finetti $\mathbb{D}^{1} \cdot$ Chiara Cassioli $\mathbb{D}^{1} \cdot$ Valentina Cianfanelli $\mathbb{D}^{2} \cdot$ Anna Onnis $\mathbb{D}^{1} \cdot$ Eugenio Paccagnini $^{1} \cdot$ \\ Anna Kabanova ${ }^{1} \cdot$ Cosima T. Baldari $^{1}$
}

Received: 31 October 2018 / Revised: 16 April 2019 / Accepted: 14 May 2019 / Published online: 29 May 2019

(c) ADMC Associazione Differenziamento e Morte Cellulare 2019

\begin{abstract}
The assembly and function of the primary cilium depends on multimolecular intraflagellar transport (IFT) complexes that shuttle their cargo along the axonemal microtubules through their interaction with molecular motors. The IFT system has been moreover recently implicated in a reciprocal interplay between autophagy and ciliogenesis. We have previously reported that IFT20 and other components of the IFT complexes participate in the assembly of the immune synapse in the non-ciliated $\mathrm{T}$ cell, suggesting that other cellular processes regulated by the IFT system in ciliated cells, including autophagy, may be shared by cells lacking a cilium. Starting from the observation of a defect in autophagic clearance and an accumulation of lipid droplets in IFT20-deficient T cells, we show that IFT20 is required for lysosome biogenesis and function by controlling the lysosomal targeting of acid hydrolases. This function involves its ability to regulate the retrograde traffic of the cation-independent mannose-6-phosphate receptor (CI-MPR) to the trans-Golgi network, which is achieved by coupling recycling CI-MPRs to the microtubule motor dynein. Consistent with the lysosomal defect, an upregulation of the TFEB-dependent expression of the lysosomal gene network can be observed in IFT20-deficient cells, which is associated with defective tonic T-cell antigen receptor signaling and mTOR activity. We additionally show that the lysosome-related function of IFT20 extends to non-ciliated cells other than T cells, as well as to ciliated cells. Our findings provide the first evidence that a component of the IFT system that controls ciliogenesis is implicated in the biogenesis of lysosomes.
\end{abstract}

\section{Introduction}

The assembly and signaling function of the primary cilium is orchestrated by multimolecular intraflagellar transport (IFT) complexes that associate with microtubule motors to allow for the bidirectional transport of soluble proteins and

\section{Edited by S. Kumar}

Supplementary information The online version of this article (https:// doi.org/10.1038/s41418-019-0357-y) contains supplementary material, which is available to authorized users.

$\triangle$ Francesca Finetti

finetti4@unisi.it

$\triangle$ Cosima T. Baldari

cosima.baldari@unisi.it

1 Department of Life Sciences, University of Siena, Via Aldo Moro 2, 53100 Siena, Italy

2 Cell Stress and Survival Unit, Danish Cancer Society Research Center, 2100 Copenhagen, Denmark membrane receptors to and from the cilium [1-3]. We previously reported that the IFT complex component, IFT20, cooperates with other IFT proteins to promote immune synapse (IS) [4, 5] assembly in the non-ciliated $\mathrm{T}$ cell by controlling polarized recycling to the synaptic membrane of endosomal T-cell antigen receptors (TCR), transferrin receptors, and the transmembrane adaptor linker for activation of $\mathrm{T}$ cells (LAT) [6-8]. As a result, TCR signaling and T-cell activation are impaired in IFT20depleted T cells [4]. Moreover, $\mathrm{CD}^{+}{ }^{+} \mathrm{T}$-cell proliferation and differentiation to helper $\mathrm{T}$ cells were found to be defective both ex vivo and in vivo in a $\mathrm{T}$ cell-specific conditional IFT20 knockout mouse [8]. In addition to revealing a cilium-independent function of the IFT system in $\mathrm{T}$ cells, our findings provided experimental support to the hypothesis of a vesicular trafficking-related role for IFT [9]. This function was recently underscored in ciliated cells with the implication of IFT20 in the vesicular transport of the phagophore elongation complex component ATG16L from the Golgi apparatus to the ciliary base [10]. 
The role of IFT20 in endosomal trafficking in T cells suggests that processes regulated by the IFT system in ciliated cells, including autophagy, may be shared by cells lacking a cilium. Starting from the observation of an autophagy defect in IFT20-deficient T cells, here, we show that IFT20 indirectly controls the latest stage of this process, namely cargo degradation in autolysosomes, by participating in the biogenesis and function of lysosomes. We demonstrate that IFT20 regulates the mannose-6phosphate receptor (CI-MPR)-mediated lysosomal targeting of acid hydrolases by coupling endosomal CIMPRs to dynein for retrograde transport to the trans-Golgi network (TGN). This function is shared by non-ciliated and ciliated cells. Our results unveil a new function of this ciliary protein in the regulation of a fundamental cellular process, and underscore its central role in intracellular membrane trafficking.

\section{Materials and methods}

\section{Cells, plasmids, and transfections}

Cells included stable control and IFT20KD Jurkat T-cell lines, generated as previously described [6] using two pGP676.13 plasmid constructs containing the complementary oligonucleotides corresponding to the coding region of human IFT20 or the respective empty vectors [11]. The same constructs were used to generate stable control and IFT20KD cell lines using as recipients the human MEC1 B-cell line [12]. Primary $\mathrm{T}$ cells were purified from peripheral blood of healthy donors using RosetteSept ${ }^{\mathrm{TM}}$ Human $\mathrm{T}$ cell Enrichment Cocktail (\#15061, STEMCELL Technologies) followed by Ficoll gradient centrifugation. $\mathrm{T}$-cell blasts were generated from purified human $\mathrm{T}$ cells activated for $48 \mathrm{~h}$ with Dynabead ${ }^{\circledR}$ Human T-Activator CD3/CD28 (\#111.31D, ThermoFisher Scientific) according to the manufacturer's instructions. Human primary $\mathrm{T}$ cells and human fibroblasts immortalized with hTERT (ATCC ${ }^{\circledR}$ CRL-4001 ${ }^{\mathrm{TM}}$ ) were knocked out for IFT20 expression by CRISPR-Cas 9 gene-editing technology.

Cell lines and human T-cell blasts were grown at $37^{\circ} \mathrm{C}$ in the presence of 5\% $\mathrm{CO}_{2}$, in RPMI 1640 (\#R7388, SigmaAldrich) supplemented with $10 \%$ bovine calf serum (BCS, \#SH30072.03, GE Healthcare HyClone). For human primary $\mathrm{T}$ cells, $50 \mathrm{U} / \mathrm{ml}$ of recombinant human IL-2 (\#130097-745, Miltenyi Biotec) was added. Fibroblasts were grown in a 4:1 mixture of the Dulbecco's medium (\#RNBG7734, Sigma-Aldrich) and Medium 199 (\#31153 Gibco) with $0.01 \mathrm{mg} / \mathrm{ml}$ hygromycin B (\#10687010, Invitrogen) and $10 \%$ fetal bovine serum (FBS, \#ECS0180L, Euroclone).
Jurkat $\mathrm{T}$ cells were transiently transfected using DEAEdextran or by electroporation with the plasmid constructs IFT20-GFP [11], TFEB-GFP (kindly provided by D. Medina), pCMV-EGFP-C3-Rab11 (kindly provided by M. Zerial), or pmRFP-EGFP-rLC3 [13], and analyzed $24 \mathrm{~h}$ post transfection. Human IFT20-specific esiRNAs (Mission human IFT20 esiRNA, \#EHU123981) and a scrambled esiRNA control Renilla luciferase (Mission RLUCRenilla luciferase-esiRNA, \#EHURLUC) (Sigma-Aldrich, Milan, Italy) were transfected by electroporation. To improve the transfection efficiency, the same transfection procedure was repeated $24 \mathrm{~h}$ post transfection, and assays were carried out after further $24 \mathrm{~h}$. Human primary T cells and hTERT fibroblasts were transfected with Cas9:gRNA ribonucleoprotein (RNP) complexes (Cas9:gRNA ratio 5:3, $\mu \mathrm{g})$ using the Human T cell Nucleofector Kit (\#VPA-1002, Amaxa Biosystem) and Lipofectamine ${ }^{\mathrm{TM}}$ CRISPRMAX ${ }^{\mathrm{TM}}$ Cas9 Transfection Reagent (\#CMAX00015, Invitrogen), respectively. DNA templates for sgRNAs synthesis were prepared by PCR amplification using the primers listed in Table S1 and pSpCas9(BB)-2A-GFP (\#48138, Addgene; deposited by Fengh Zhang) [14] as a template. sgRNAs were transcribed in vitro using HiScribe ${ }^{\mathrm{TM}} \mathrm{T} 7 \mathrm{High}$ Yield RNA Synthesis Kit (\#E2040S, NEB) and purified with RNA Clean \& Concentrator ${ }^{\mathrm{TM}}$ (\#R1017, Zymo Research). Cells were analyzed for genome editing at least $72 \mathrm{~h}$ post transfection, and assays were carried out 7 days post transfection.

\section{Antibodies and reagents}

Polyclonal anti-IFT20 antibodies were previously described [15]. All primary commercial antibodies used in this work are listed in Table S2, together with information about the dilutions used for immunoblotting and immunofluorescence. Secondary peroxidase-labeled antibodies were from Amersham Biosciences. Alexa Fluor 488- and 555labeled secondary Abs were from ThermoFisher Scientific (anti-mouse 488, \#A11001; anti-rabbit 488, \#A11008; antimouse 555, \#A21422; anti-rabbit 555, \#A21428). Other reagents included chloroquine (C6628, Sigma-Aldrich), leupeptin (L8511, Sigma-Aldrich), pepstatin A (\#P4265, Sigma-Aldrich), ciliobrevin D (\#250401, Merck Millipore), BODIPYTM 493/503 (4,4-difluoro-1,3,5,7,8-pentamethyl-4bora-3a,4a-diaza-s-indacene, \#D3922, Molecular Probes), LysoTracker $^{\mathrm{TM}}$ red DND-99 (\#L7528, Invitrogen), DQ ${ }^{\mathrm{TM}}$ Green BSA (\#D12050, Invitrogen), and Magic Red Cathepsin B substrate (\#937, Immunochemistry Technologies). The recombinant fusion proteins, GST-IFT20 as well as control GST, were affinity purified on GSH-Sepharose (GE Healthcare) from bacterial cultures incubated with $0.25 \mathrm{mM}$ isopropyl- $\beta$-D-thiogalactopyranoside for $4 \mathrm{~h}$ at 37 ${ }^{\circ} \mathrm{C}$ and lysed by sonication in PBS $1 \%$ Triton $\mathrm{X}-100$. To 
assess the role of the kinases ERK and mTOR on TFEB activity, cells were treated for $16 \mathrm{~h}$ with $20 \mu \mathrm{M}$ PD098059 (\#P215, Sigma-Aldrich) or $250 \mathrm{nM}$ Torin (\#4247, Tocris Bioscience).

\section{Autophagic flux measurement, apoptosis measurement, and lysosome purification}

To monitor autophagic flux, cells $\left(1 \times 10^{6} /\right.$ sample $)$ were either left untreated or treated with $40 \mu \mathrm{M}$ chloroquine in RPMI 1640 added with $10 \%$ FCS or Earle's balanced salt solution (EBSS, Sigma-Aldrich) for $1 \mathrm{~h}$ at $37^{\circ} \mathrm{C}$. To inhibit acid proteases, cells were untreated or treated with $150 \mu \mathrm{M}$ leupeptin or $100 \mu \mathrm{M}$ pepstatin A in RPMI 1640 added with $10 \%$ FCS for $16 \mathrm{~h}$ at $37^{\circ} \mathrm{C}$. The effect of dynein inhibition was analyzed incubating the cells in the presence or absence of $50 \mu \mathrm{M}$ ciliobrevin D in RPMI 1640 $10 \% \mathrm{FCS}$ for $16 \mathrm{~h}$ at $37^{\circ} \mathrm{C}$. After the treatments, cells were harvested and lysed in $1 \%$ Triton X-100 in $20 \mathrm{mM}$ Tris- $\mathrm{HCl} \mathrm{pH} 8.0,150 \mathrm{mM} \mathrm{NaCl}$ in the presence of a protease inhibitor cocktail (\#539134, Calbiochem) and the phosphatase inhibitor sodium vanadate (\#S6508, Sigma-Aldrich), and processed for immunoblotting with anti-LC3II antibodies. The densitometric analysis of LC3-II and actin was carried out using ImageJ. The difference in LC3-II levels, normalized to actin, between chloroquine-treated and untreated cells was reported as autophagy flux in the graphs. Alternatively, cells were transiently transfected with the vector pmRFP-EGFPrLC3 [13] and analyzed $24 \mathrm{~h}$ post transfection. The fluorescent dots of GFP-LC3 and RFP-LC3, either as such or following staining with anti-GFP and anti-RFP antibodies, were measured on median optical sections using ImageJ.

Apoptosis was measured by flow-cytometric analysis of cells incubated for $24 \mathrm{~h}$ in low-serum $(0.5 \%)$ medium and stained with FITC-labeled annexin $\mathrm{V}$ and propidium iodide using FITC Annexin V Apoptosis Detection Kit with PI (\#640914, BioLegend) and following the manufacturer's instructions. Cells were analyzed using a GUAVA flow cytometer (Millipore), gating on Annexin V-positive and propidium iodide-negative cells, which were considered as early apoptotic.

Purification of lysosomes was performed from $10^{8}$ cells using the Lysosome Enrichment Kit for Tissue and Cultured Cells (89839, ThermoFisher Scientific) following the manufacturer's instructions. Eight fractions were collected from the top of the OptiPrep gradients tube and analyzed by SDS-PAGE to determine the fractions enriched in lysosomes (Fig. S1). Equal amounts of proteins from the pool of the fractions enriched in LAMP-1 were analyzed for the presence of lysosomal hydrolases.

\section{Immunoblotting, immunoprecipitation, and in vitro- binding assays}

For immunoprecipitation experiments, cells $\left(5 \times 10^{7} / \mathrm{sam}-\right.$ ple) were pelleted, washed twice in ice-cold PBS and lysed in $500 \mu \mathrm{l}$ of $0.5 \%$ Triton $\mathrm{X}-100$ in $20 \mathrm{mM}$ Tris- $\mathrm{HCl} \mathrm{pH} \mathrm{8.0,}$ $150 \mathrm{mM} \mathrm{NaCl}$ in the presence of a protease inhibitor cocktail and phosphatase inhibitor sodium vanadate. Postnuclear supernatants were immunoprecipitated for $2 \mathrm{~h}$ using $2 \mu \mathrm{g}$ of rabbit anti-IFT20 antibody (\#13615-1-AP, Proteintech) or mouse anti-dynein mAb (MAB1618, Merck Millipore), and $3 \mathrm{mg} / \mathrm{sample}$ protein-A-Sepharose (PAS; \#17-0780-01, GE Healthcare) (Amersham). Before immunoprecipitation, post-nuclear supernatants were precleared for $1 \mathrm{~h}$ with the same amount of PAS. PAS-antibody complexes as well as PAS controls were pelleted, washed $4 \times$ with $1 \mathrm{ml}$ of $0.5 \%$ Triton X-100 lysis buffer, then resuspended in $15 \mu \mathrm{l}$ of Laemmli buffer (\#B0007, Life Technologies), boiled for $5 \mathrm{~min}$ and subjected to SDSPAGE. All gels included a sample of the lysates used for the immunoprecipitations (10 $\mu \mathrm{g} / \mathrm{sample})$. Quantification was carried out using the BCA Assay kit (\#EMP014500, EuroClone).

In vitro-binding assays were carried using recombinant GST/GST-IFT20 on GSH-Sepharose precleared postnuclear supernatants from $10^{8}$ cells/sample lysed in $0.5 \%$ Triton $\mathrm{X}-100$ in the presence of protease inhibitors.

Immunoblotting was carried out using peroxidaselabeled secondary Ig and a chemiluminescence detection kit (\#34078, Pierce Rockford). Stripping was carried out using ReBlot Plus Mild Antibody Stripping Solution, $10 \times$ (\#2502, Merck Millipore). Blots were scanned using a laser densitometer (Duoscan T2500; Agfa), and the densitometric analysis was carried out using ImageJ 1.46r (National Institutes of Health, USA).

\section{CI-MPR recycling, immunofluorescence microscopy, and colocalization analyses}

To analyze recycled CI-MPR, cells were incubated with saturating concentrations of anti-CI-MPR $\mathrm{mAb}$ at $37^{\circ} \mathrm{C}$ for 30 or $60 \mathrm{~min}$ or $4 \mathrm{~h}$. Subsequently, cells were plated onto poly-L-lysine-coated wells and fixed by immersion in methanol for $10 \mathrm{~min}$ at $-20^{\circ} \mathrm{C}$. Following fixation, samples were washed for $5 \mathrm{~min}$ in PBS and incubated with fluorochrome-labeled secondary antibodies for $45 \mathrm{~min}$ at RT. For LC3B labeling, Jurkat cells were fixed in $4 \%$ paraformaldehyde for $10 \mathrm{~min}$ at RT, incubated $10 \mathrm{~min}$ in 50 $\mathrm{mM} \mathrm{NH}_{4} \mathrm{Cl}$, and then permeabilized in methanol for $10 \mathrm{~min}$ at $-20{ }^{\circ} \mathrm{C}$. Samples were incubated with primary antibodies overnight at $4{ }^{\circ} \mathrm{C}$, after blocking in $5 \%$ normal goat serum and $1 \%$ BSA for $1 \mathrm{~h}$. After washing in PBS, samples were incubated for $1 \mathrm{~h}$ at RT with Alexa-Fluor 488- and 
555-labeled secondary antibodies. Alternatively, cells were loaded for $10 \mathrm{~min}$ at $37^{\circ} \mathrm{C}$ with $75 \mathrm{nM}$ LysoTracker ${ }^{\mathrm{TM}}$ red DND-99 and/or $1 \mu \mathrm{M}$ BODIPYTM 493/503 in PBS or for $4 \mathrm{~h}$ at $37^{\circ} \mathrm{C}$ in complete medium with $1 \mu \mathrm{M}$ Magic Red cathepsin B substrate and analyzed by confocal microscopy. The length of cilia was measured using ImageJ on hTERT fibroblasts grown to 70-90\% confluency and serum-starved for $48 \mathrm{~h}$, followed by fixation and permeabilization by immersion in methanol for $10 \mathrm{~min}$ at $-20^{\circ} \mathrm{C}$ and staining with an anti-acetylated tubulin mAb.

Confocal microscopy was carried out on a Zeiss LSM700 using a $\times 63$ or $\times 40$ objective. Z series of optical sections were performed at $0.5-\mu \mathrm{m}$ increments. Images were acquired with pinholes opened to obtain $0.8-\mu$ m-thick sections. Detectors were set to detect an optimal signal below the saturation limits. Images were processed with Zen 2009 image software (Carl Zeiss, Jena, Germany).

The colocalization analyses were performed on median optical sections using ImageJ and the JACoP plug-in to determine Manders' coefficient M1 $(0=$ no colocalization; $1=100 \%$ colocalization), as previously described [7]. The CI-MPR dispersion was quantified as percentage of cells where more than $40 \%$ of CI-MPR ${ }^{+}$vesicles are distant more than $2.5 \mu \mathrm{m}$ from the centrosome in individual medial confocal sections. The number and size of vesicles positive for each marker were determined in individual medial confocal sections using ImageJ ("Analyze particles" tool). The mean volume was analyzed on $\mathrm{z}$ series of optical sections performed at $0.8-\mu \mathrm{m}$ increments using ImageJ and the Object counter 3D, and indicated as mean number of LAMP- ${ }^{+}{ }^{+}$vesicle voxels/cell [16].

\section{Electron microscopy}

Jurkat cell pellets were fixed in $2.5 \%$ glutaraldehyde diluted in PBS for $1 \mathrm{~h}$ at $4{ }^{\circ} \mathrm{C}$, washed in buffer overnight, and then postfixed in $1 \%$ osmium tetroxide for $1 \mathrm{~h}$ at $4{ }^{\circ} \mathrm{C}$. Following one further rinse in buffer, samples were dehydrated with a graded series of ethanols and embedded in Epon. Thin sections were obtained with a Reichert Ultracut E ultramicrotome and recovered on 100 mesh copper grids. Sections were routinely stained with uranyl acetate and lead citrate and observed with a FEI Tecnai G2 Spirit transmission electron microscope at an electron accelerating voltage of $100 \mathrm{kV}$. The lipid droplet diameter was measured using ImageJ (“Analyze particles” tool).

\section{RNA purification and RT-qPCR}

RNA was extracted, reverse transcribed, and analyzed by RT-qPCR, performed in triplicate on each cDNA in 96-well optical PCR plates (Sarstedt), as described previously [7]. HPRT1 was used as a housekeeping gene to normalize transcript levels. The primers used to amplify the cDNA fragments corresponding to human transcripts are listed in Table S1.

\section{Analysis of $\mathrm{Cl}-\mathrm{MPR}$ expression and internalization}

Flow-cytometry analysis of CI-MPR expression was performed using anti-CI-MPR Ab (1:200, ab32815, Abcam) followed by secondary anti-rabbit Ig 488-labeled antibody. To monitor CI-MPR internalization, cells were incubated on ice with anti-CI-MPR Ab for $30 \mathrm{~min}$, washed (time 0), and shifted to $37^{\circ} \mathrm{C}$ for 15 or $30 \mathrm{~min}$. The levels of surface receptor were determined at the different time points by flow cytometry by staining the cells with secondary antirabbit Ig 488-labeled antibody. The data are presented as percentage of CI-MPR internalization calculated by setting the time 0 value as $100 \%$. Flow cytometry was carried out using a GUAVA flow cytometer (Millipore).

\section{Statistical analysis}

Mean values, standard deviation values, and statistical significance were calculated using GraphPad (Prism Software). Values with normal distribution were analyzed with Student's $t$ test (paired or unpaired), one sample $t$ test (theoretical mean =1) and one-way ANOVA followed by Tukey post hoc test (for multiple comparisons); values without Gaussian distribution were analyzed with Mann-Withney test or Kruskal-Wallis test followed by Dunn's test (for multiple comparisons) using GraphPad (Prism Software). A $P$-value $<0.05$ was considered as statistically significant.

\section{Results}

\section{IFT20 is required for T-cell autophagy}

To address the role of IFT20 in T-cell autophagy, we measured the autophagic flux in a Jurkat T-cell transfectant stably knocked down for IFT20 expression (IFT20KD) and the respective control (Fig. 1a). Cells were either untreated or treated with the lysosomal inhibitor chloroquine, which blocks the degradation of the cleaved and lipidated form of LC3, LC3-II. Immunoblot analysis with anti-LC3 antibodies showed decreased autophagic flux in IFT20KD cells compared with the control transfectant both in basal and starvation (EBSS) conditions (Fig. 1a). Similar results were obtained using the tandem fluorescent-tagged LC3 (mRFPEGFP-LC3), which allows to monitor autophagic flux based on different $\mathrm{pH}$ stability of EGFP and mRFP fluorescent proteins [13] (Fig. 1b). Hence, similar to ciliated cells, IFT20 participates in autophagy in the non-ciliated $\mathrm{T}$ cell. 
a
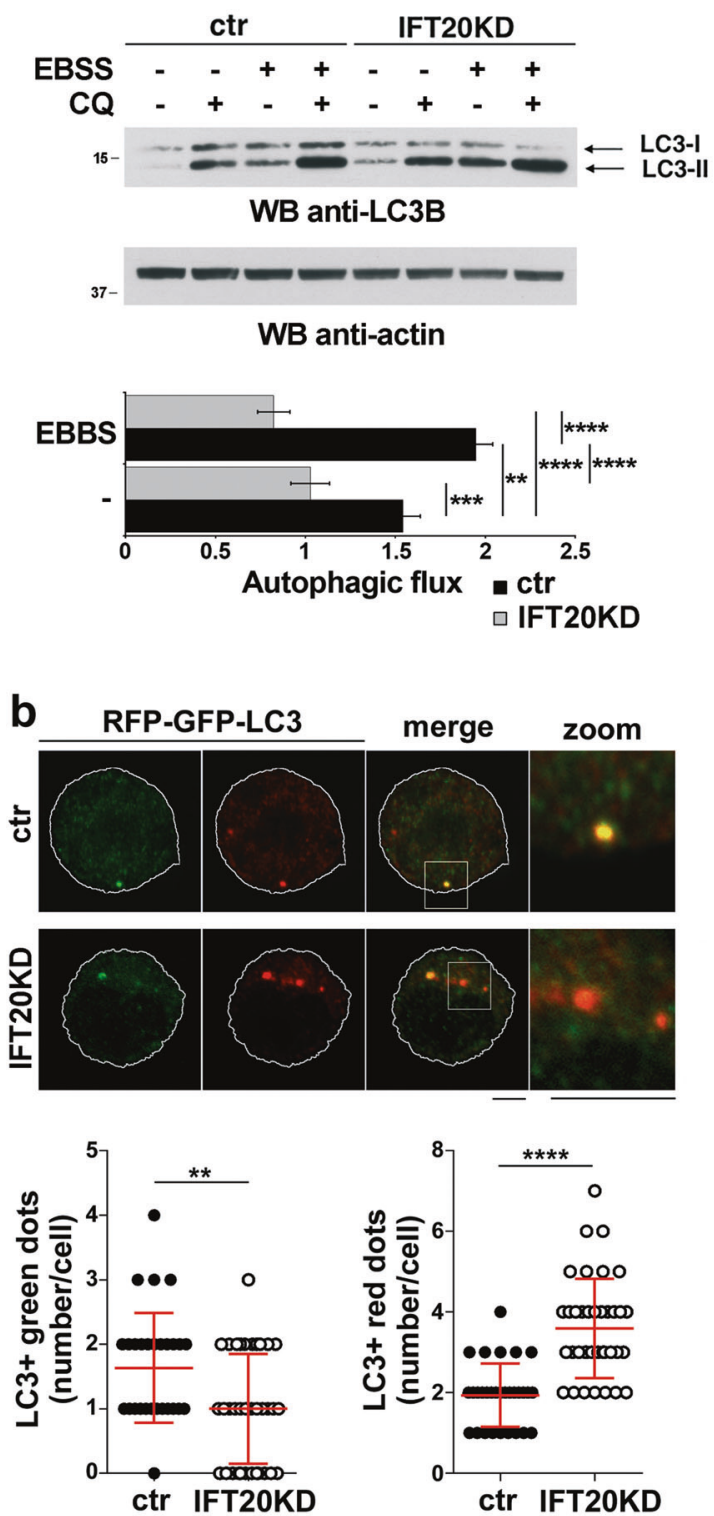

C

15-

WB anti-IFT20

37- -

WB anti-actin

$$
\begin{aligned}
& \begin{array}{ll}
1 & 0.19
\end{array} \\
& +1-+1- \\
& \begin{array}{ll}
0 & 0.02
\end{array} \\
& { }^{* * *} p<0.001
\end{aligned}
$$

d
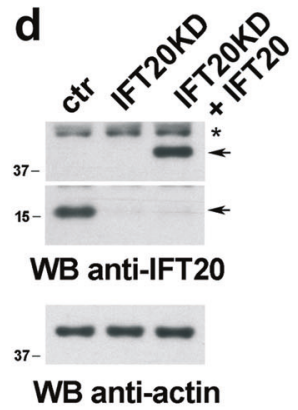

WB anti-actin

$\begin{array}{lll}1 & 0.12 & 1.19\end{array}$

$+/-\quad+1-\quad+1-$

$\begin{array}{lll}0 & 0.03 & 0.22\end{array}$

${ }_{* * * *} p<0.0001$ ctr vs IFT2OKD;

IFT2OKD vs IFT20KD+IFT20

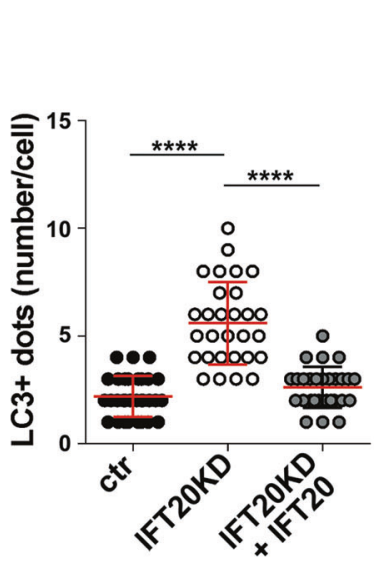

e
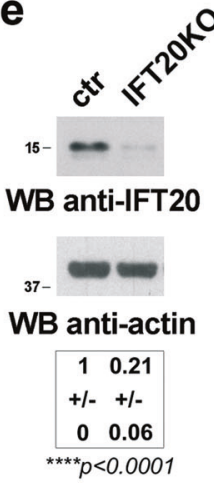

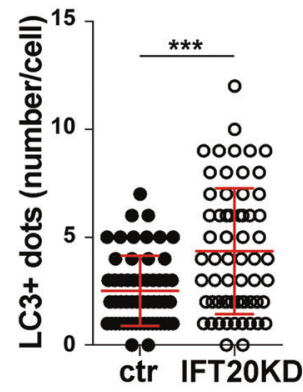

ctr IFT20KD 
Fig. 1 IFT20 is required for T cell autophagy. a Immunoblot analysis of the autophagy marker LC3B in lysates of ctr and IFT20KD Jurkat cells, either untreated or treated for $30 \mathrm{~min}$ with Earle's starvation buffer (EBSS) in the presence or absence of chloroquine (CQ, $40 \mu \mathrm{M})$. The migration of molecular mass markers is indicated. The histogram below shows the autophagic flux in ctr and IFT20KD EBSS-untreated or EBSS-treated cells, calculated as the difference in the levels of LC3II (normalized to actin) between CQ-treated and CQ-untreated samples for each transfectant and condition (mean fold $\pm \mathrm{SD}$; normalized to vehicle control value in ctr cells set as 1, one-way ANOVA; $n=3$ ). $\mathbf{b}$ Quantification of the number of $\mathrm{GFP}^{+}$and $\mathrm{RFP}^{+}$dots in ctr and IFT20KD Jurkat cells transiently transfected with the pmRFPEGFP-rLC3 construct and labeled with anti-GFP and anti-RFP antibodies ( $\geq 10$ cells/sample, $n=3$; Mann-Whitney test). Representative images (median optical sections) are shown. Size bar, $5 \mu \mathrm{m}$. c-e Immunofluorescence analysis of LC3 in ctr and IFT20KD Jurkat cells (c), IFT20KD Jurkat cells transiently transfected with a construct encoding GFP-tagged IFT20 or control and IFT20KD cells transiently transfected with the respective GFP vector (d), or primary ctr and IFT20KO $\mathrm{T}$ cells (e). The graphs show the quantification of the number of $\mathrm{LC}^{+}$dots/cell (mean $\pm \mathrm{SD}$; (c, e) Mann-Whitney test (d) Kruskal-Wallis test). At least 30 cells from three independent experiments were analyzed. A representative anti-IFT20 immunoblot of the cells used is shown in each panel. Actin was used as loading control. The migration of molecular mass markers is indicated. The quantification of the relative protein expression normalized to actin (mean fold $\pm \mathrm{SD}$; ctr value $=1)$ is reported below each blot $(n \geq 3$; (c, e) one sample $t$ test, (d) ANOVA one-way test). f Immunofluorescence analysis of LC3 and LAMP-1 in ctr and IFT20KD Jurkat cells. Quantification of the number of autolysosomes $\left(\mathrm{LC}^{+}\right.$ LAMP-1 ${ }^{+}$vesicle number/cell) in ctr and IFT20KD Jurkat cells (mean $\pm \mathrm{SD}$; Mann-Whitney test). At least 25 cells from three independent experiments were analyzed. Representative images (medial optical sections) are shown. Size bar: $5 \mu \mathrm{m} .{ }^{* *} P<0.01$; $* * * P<0.001 ; * * * * P<0.0001$

\section{IFT20 is required for autolysosome function}

While the autophagic flux was impaired in IFT20KD $\mathrm{T}$ cells, the basal LC3-II levels were higher compared with control cells (fold increase $1.49 \pm 0.05$ w/o EBSS, $1.87 \pm$ 0.06 w/ EBSS) (Fig. 1a). Consistently, immunofluorescence analysis showed that IFT20KD cells displayed higher numbers of $\mathrm{LC}^{+}$dots compared with controls under steady state (Fig. 1c). This alteration was rescued by reconstituting IFT20 expression (Fig. 1d). The results were confirmed on Jurkat cells (Fig. S2a) transiently knocked down for IFT20 expression by RNA interference and validated on primary human IFT20KO T cells obtained by CRISPR-Cas9-based gene editing (Fig. 1e; Fig. S3a).

LC3-II is degraded upon autophagosome-lysosome fusion, and its accumulation associates with defective autolysosome function [17-19]. To investigate whether IFT20 impacts on autolysosome function, we carried out a colocalization analysis of LC3 and the lysosomal marker LAMP-1. IFT20 depletion resulted in a significant increase in the number of autolysosomes $\left(\mathrm{LC}^{+}{ }^{+} \mathrm{LAMP}^{+}\right.$vesicles) (Fig. 1f). This indicates that autophagosome fusion with lysosomes occurs normally in IFT20KD cells but that the resulting autolysosomes are dysfunctional, accounting for the LC3-II accumulation.

Transmission electron microscopy revealed an accumulation of lipid droplets (LD) [20] in IFT20KD cells (Fig. 2a), a feature also associated with defective autophagy [21]. The average LD size was smaller in IFT20-deficient cells (Fig. 2a), as confirmed by imaging ctr after loading with the fluorescent LD probe BODIPY (Fig. 2b). Co-staining BODIPY-loaded cells with the lysosomal probe Lysotracker Red showed an increased colocalization of LDs with lysosomes in IFT20KD cells (Fig. 2c), suggesting that LDs fail to be degraded, consistent with impaired autolysosome function.

\section{IFT20 regulates the TFEB-dependent lysosome biogenesis program}

The autolysosome abnormalities observed in IFT20KD $\mathrm{T}$ cells downstream of autolysosome generation suggest a lysosome defect. We noticed that the average size of lysosomes (LAMP-1-positive vesicles) was larger in IFT20KD cells compared with controls, which was paralleled by an increase in the total cellular volume of lysosomes and a reduction in their number (Fig. 3a; Fig. S2b). The defect was rescued by restoring IFT20 expression (Fig. 3b). This phenotype is consistent with a defect in lysosome biogenesis, a process orchestrated by the CLEAR gene network coordinated by the transcription factor TFEB [22]. Indeed, IFT20KD cells displayed higher levels of LAMP-1, which is regulated by TFEB (Fig. 3c). A compensatory upregulation of genes encoding other components of the CLEAR network, namely the lysosomal membrane protein LAMP2, the GTPase Rab7, the proteases cathepsins B (catB) and $\mathrm{D}$ (catD), the glycosaminoglycan-degrading enzyme hexosaminidase $\mathrm{B}$ (hexB), and the lysosomal acid lipase (LAL), was observed in IFT20-deficient T cells (Fig. 3d). An upregulation of TFEB, on which expression of these genes depends [23, 24], was also observed (Fig. 3d; Fig. S2c). These alterations were rescued by reconstituting IFT20 expression (Fig. 3e). Similar results were obtained on primary IFT20KO cells (Fig. 3f; Fig. S3b).

Of note, TFEB activity was enhanced in IFT20KD cells, as assessed by imaging nuclear TFEB in control and IFT20KD cells transfected with GFP-tagged TFEB (Fig. 4a). TFEB nuclear translocation is negatively controlled by mTOR and/or ERK-dependent serine phosphorylation [25, 26]. Immunoblot analysis with phosphospecific antibodies showed that the basal levels of active P-mTOR and P-ERK1/2 were decreased in IFT20KD cells (Fig. 4b), potentially accounting for the enhanced nuclear localization of TFEB in these cells. To dissect the respective role of these kinases in TFEB activity, control cells were treated with the ERK inhibitor PD098059 or the mTOR inhibitor Torin1. mTOR inhibition, but not ERK inhibition, 
Fig. 2 IFT20 is required for lipid droplet breakdown. a Transmission electron microscopy of ctr and IFT20KD Jurkat cells. Lysosomes (Ly) and lipid droplets (LD) are indicated. The graph shows the quantification of the diameter of lipidic droplets in ctr and IFT20KD Jurkat cells $(n=3)$. Size bar: $2 \mu \mathrm{m}$. b Fluorescence analysis of ctr and IFT20KD Jurkat cells loaded with the LD marker BODIPY.

Representative images (medial optical sections) are shown on the left. Size bar: $5 \mu \mathrm{m}$. The graphs show the quantification of the number of BODIPY ${ }^{+}$ dots/cell (middle) and the average size of BODIPY $^{+}$dots (right) (mean $\pm \mathrm{SD})$. At least 21 cells from three independent experiments were analyzed. c Immunofluorescence analysis of ctr and IFT20KD Jurkat cells co-labeled with BODIPY and Lysotracker Red (LyTr).

Representative images (medial optical sections) are shown on the left. Size bar: $5 \mu \mathrm{m}$. The graph shows the quantification of the colocalization of BODIPY and LyTr, expressed as

Mander's coefficient (mean \pm SD). At least 28 cells from three independent experiments were analyzed. $* P<0.05 ; * * * P<$ 0.001 (Mann-Whitney test) a

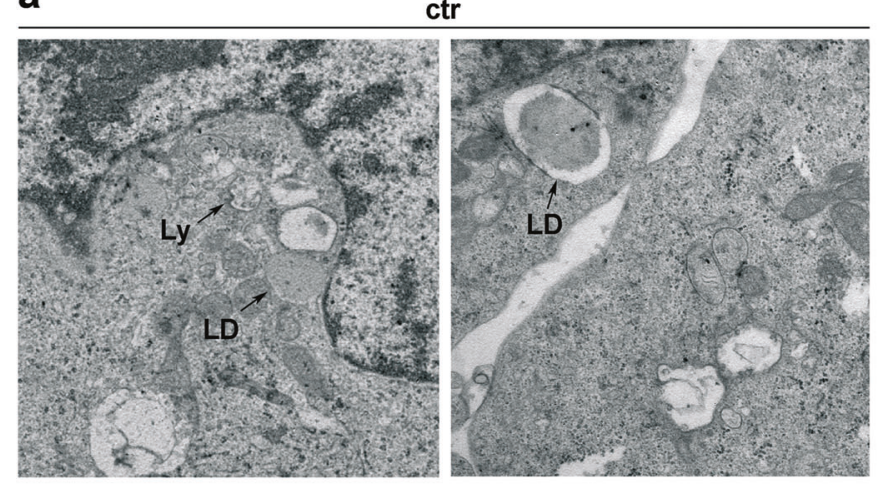

IFT20KD
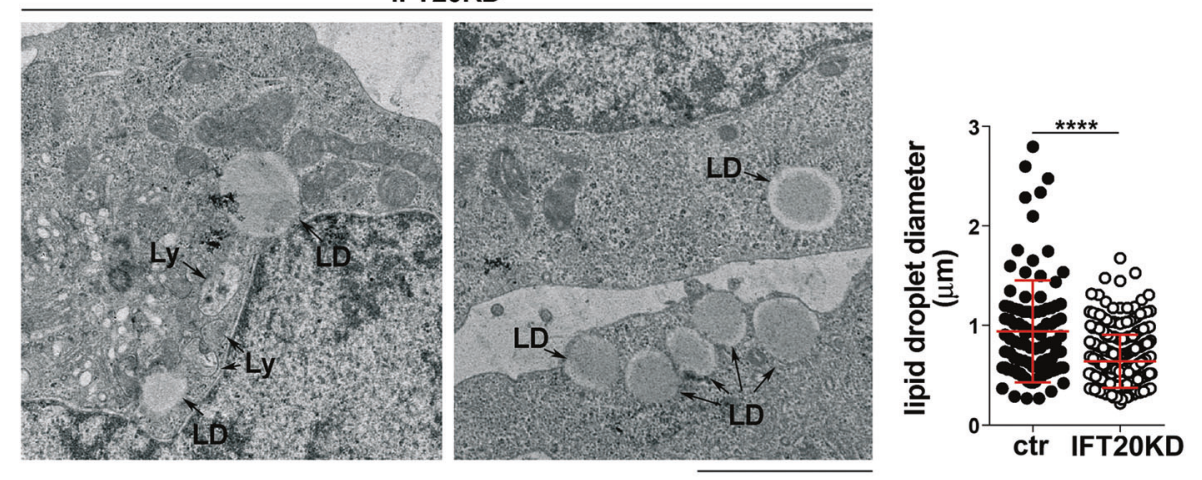

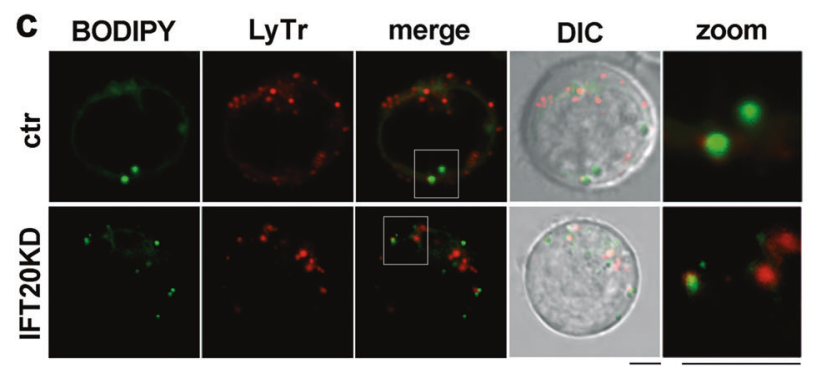

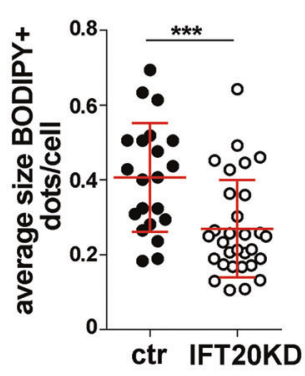
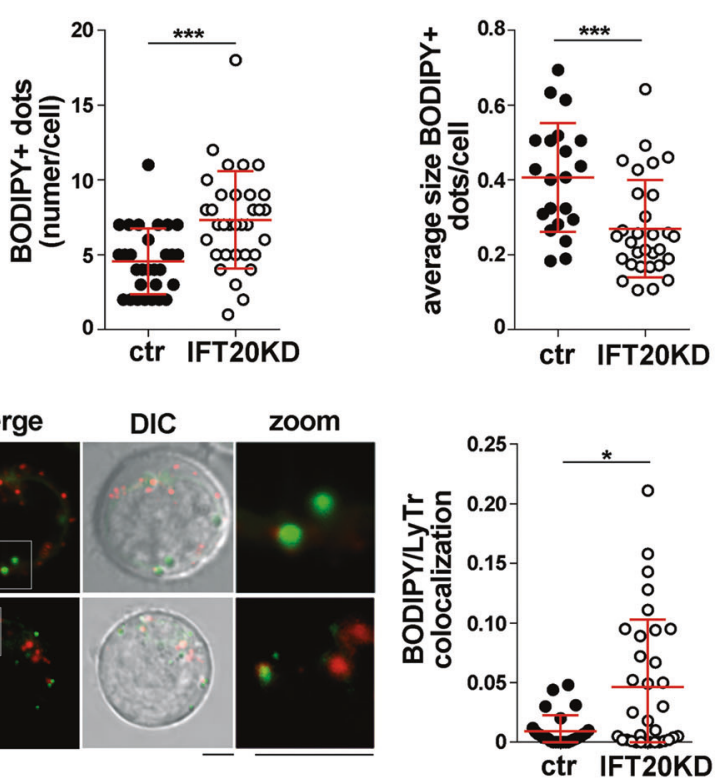

reproduced the effects of IFT20 knockdown on the TFEBdependent transcription of lysosomal genes (Fig. 4c, d). These results indicate that the TFEB-dependent compensatory enhancement of lysosome biogenesis observed in IFT20KD cells can be selectively ascribed to the decrease in mTOR signaling.

The lower basal ERK and mTOR phosphorylation in IFT20KD cells suggests that IFT20 may participate in tonic signaling by the TCR [27]. To test this hypothesis, we analyzed the activation status of TCR-proximal signaling components upstream of these kinases. The basal levels of ZAP-70 and LAT phosphorylation, which correlates with their activation, were lower in IFT20KD cells compared with controls. Conversely, Lck phosphorylation on its inhibitory C-terminal tyrosine was higher in IFT20KD cells (Fig. 4e). In addition, lower mRNA levels of two specific tonic signaling markers, the orphan receptor Nur77 and CD5 [27], were observed in these cells (Fig. 4f). 

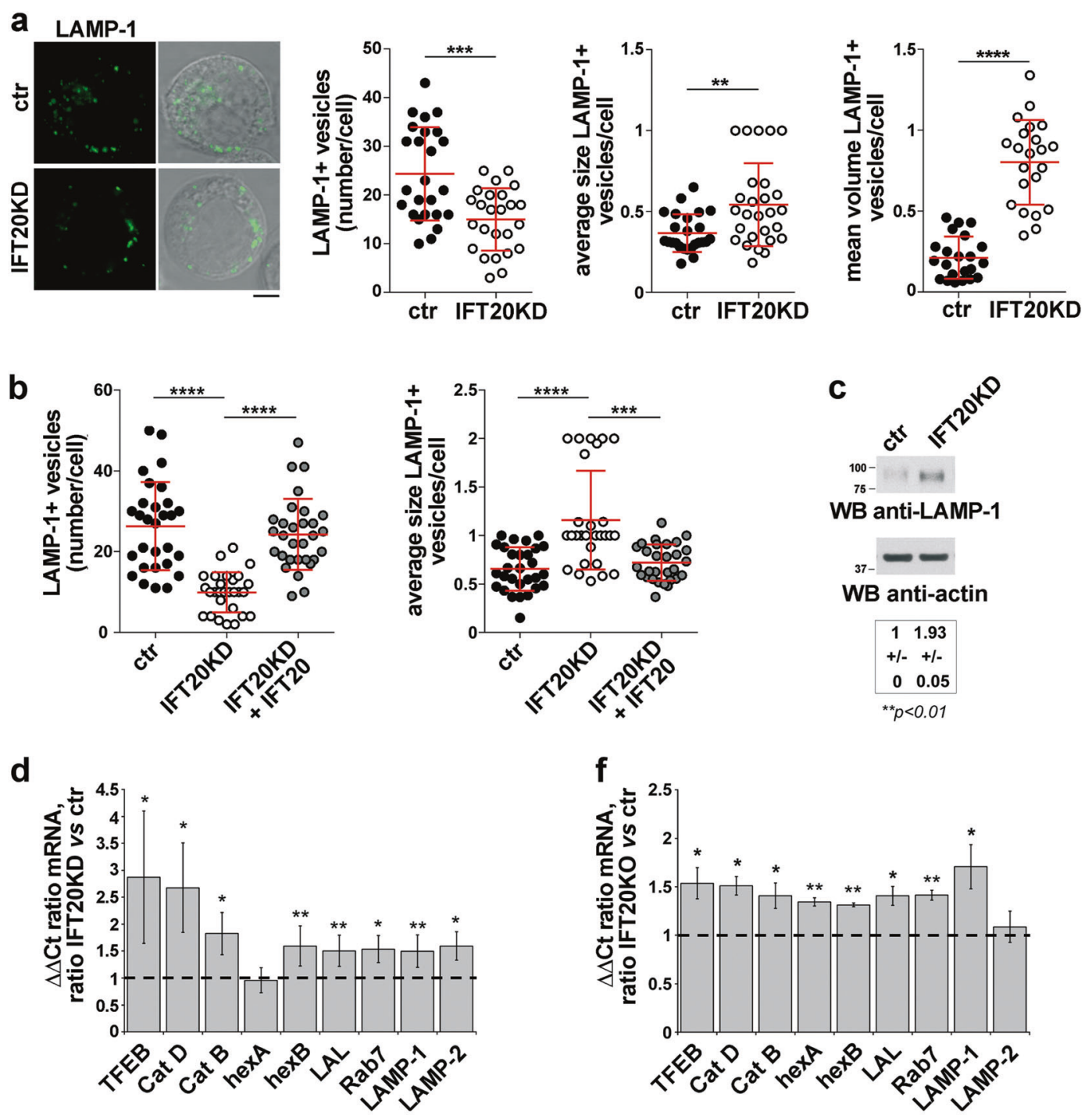

e

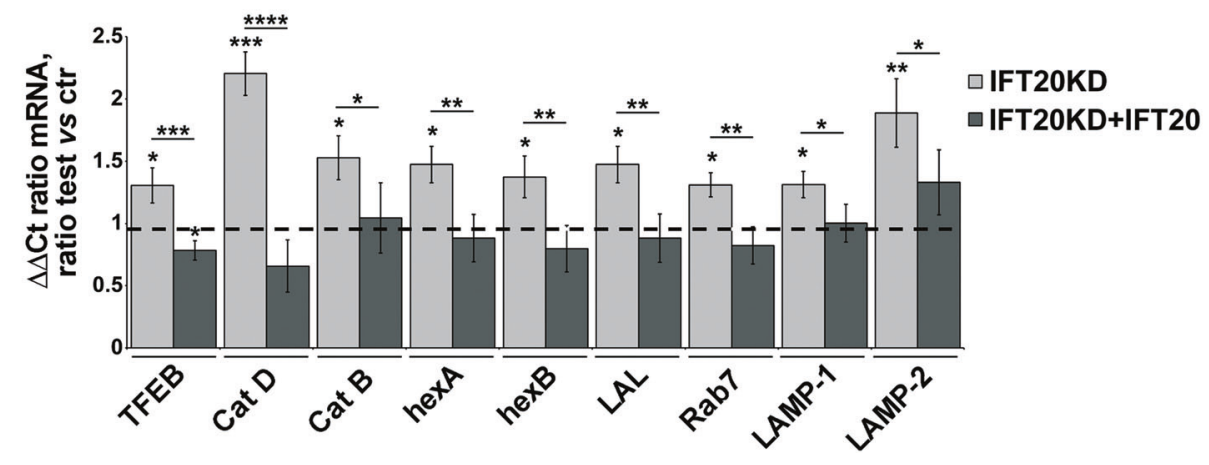

Consistent with the implication of tonic signaling in T-cell homeostasis [27], survival was impaired in IFT20deficient cells (Fig. 4g). Hence the IFT20-mediated regulation of tonic TCR signaling may also explain the deregulation of mTOR activity and the resulting TFEBdependent activation of the CLEAR network in IFT20KD $\mathrm{T}$ cells.

\section{IFT20 is required for the traffic of acid hydrolases to} lysosomes

Imaging of Jurkat cells or primary $\mathrm{T}$ cells loaded with Lysotracker Red, a pH-sensitive lysosomal probe, showed an increase in fluorescence in IFT20-deficient cells compared with controls (Fig. 5a, b; Fig. S3c) which paralleled 
Fig. 3 IFT20 participates in lysosome biogenesis. a Immunofluorescence analysis of LAMP-1 in ctr and IFT20KD Jurkat cells. Representative images (medial optical sections) are shown (left). Size bar: $5 \mu \mathrm{m}$. The graphs show the quantification of the number of LAMP- $1^{+}$vesicles/cell (Student's $t$ test), their average size $\left(\mu \mathrm{m}^{2}\right)$ (Mann-Whitney test) and their volume (mean number of LAMP- ${ }^{+}$ vesicle voxels/cell) (Mann-Whitney test) (mean \pm SD). At least 25 cells from three independent experiments were analyzed. b Immunofluorescence analysis of LAMP-1 in IFT20KD Jurkat cells transiently transfected with a construct encoding GFP-tagged IFT20 or control and IFT20KD Jurkat cells transiently transfected with the respective GFP vector. The graphs show the quantification of the number and average size $\left(\mu \mathrm{m}^{2}\right)$ of $\mathrm{LAMP}-1^{+}$vesicles/cell (mean $\pm \mathrm{SD}$; Kruskal-Wallis test). At least 28 cells from three independent experiments were analyzed. c Immunoblot analysis of LAMP-1 in ctr and IFT20KD Jurkat T cells. Actin was used as loading control. A representative immunoblot is shown. The migration of molecular mass markers is indicated. The quantification of the relative protein expression (mean fold $\pm \mathrm{SD}$; $\operatorname{ctr}$ value $=1)$ is reported below $(n=3$; one sample $t$ test). d-f Quantitative RT-PCR analysis of TFEBregulated genes and TFEB in control and IFT20KD Jurkat T cells $(n \geq$ 4; one sample $t$ test) (d), IFT20KD Jurkat cells transiently transfected with a construct encoding GFP-tagged IFT20 or control and IFT20KD cells transiently transfected with the respective GFP vector $(n \geq 3$; oneway ANOVA) (e), or human primary control and IFT20KO T cells gene-edited by CRISPR-Cas9 technology ( $n=3$; one sample $t$ test) (f). The relative abundance of gene transcripts was determined on triplicate samples using the ddCt method and was normalized to HPRT1. The data $($ mean $\pm \mathrm{SD})$ are expressed as normalized fold expression in IFT20KD/KO vs control cells (expression in ctr cells set for each gene as 1 , dashed line). $* P<0.05 ; * * P<0.01 ; * * * P<0.001$; $* * * * P<0.0001$

the increase in the total cellular volume of lysosomes (Fig. 3a), indicating that acidification occurred normally in these cells. Conversely, flow-cytometric analysis of cells loaded with DQ-BSA, a protease substrate which becomes fluorescent when cleaved, showed that protease activity was impaired in IFT20KD cells (Fig. 5c). Similar results were obtained using as a probe Magic Red, which specifically detects catB activity (Fig. 5d and Fig. S2d; Fig. 5e and Fig. S3d for primary T cells). IFT20 reconstitution restored catB activity in IFT20KD cells (Fig. 5f).

Since lysosomal proteases are activated at the low $\mathrm{pH}$ of the lysosome lumen, which is not affected by IFT20 deficiency, this suggests that their transport might be compromised in IFT20KD cells. We initially focused on catD, a lysosomal aspartyl-endopeptidase implicated in autophagy [28]. Treatment of control cells with either the acid protease inhibitor pepstatin-A, or with leupeptin, a serine/cysteine protease inhibitor that indirectly inhibits catD maturation [29], reproduced the effects of IFT20 depletion in control cells, promoting LC3-II accumulation (Fig. S4a, b). These results suggest that IFT20 may be implicated in its traffic to lysosomes.

Pro-cathepsin D (pro-catD) is added with mannose-6phosphate (M6P) at the Golgi, acquiring the ability to bind to the M6P receptor (MPR) at the trans-Golgi network (TGN). It is then transported to the endolysosomal system where it dissociates from the MPR and becomes proteolytically activated [30]. Consistent with the mRNA analysis (Fig. 3e), an increase in the total catD was observed in IFT20KD cells (Fig. 5g), however, the levels of mature catD were lower compared with controls (Fig. 5g;Fig. S5). In addition, the proportion of processed catD was lower in IFT20KD cells (Fig. 5g), suggesting a defect in pro-catD targeting to lysosomes.

The impact of IFT20 depletion on the lysosomal localization of catD was investigated by imaging ctr and IFT20KD cells co-stained for catD and LAMP-1. Colocalization analyses showed that the catD content of lysosomes was lower in IFT20KD cells compared with controls (Fig. 5h). Moreover, immunoblot analysis of lysosomeenriched fractions showed that, despite the increase in LAMP-1, the levels of mature lysosomal catD were lower in IFT20KD cells (Fig. 5i). Similarly, hexA/hexB and LAL, which also require CI-MPRs for lysosomal transport, were present at lower levels in lysosomes from IFT20KD cells (Fig. 5i). These data account for the lysosomal accumulation of LC3-II and LDs in IFT20KD cells and indicate that IFT20 participates in the trafficking pathway that controls the lysosomal delivery of acid hydrolases.

\section{IFT20 controls the lysosomal targeting of acid hydrolases by regulating MPR traffic}

The lysosomal transport of acid hydrolases is largely controlled by the CI-MPR, a type-I $300-\mathrm{kDa}$ transmembrane protein localized mainly at the TGN and endosomes [31]. CI-MPRs are sorted at the TGN into clathrin-coated vesicles that traffic to early endosomes (EE) and then to late endosomes (LE), with a small proportion being targeted to the plasma membrane. In LEs, the low $\mathrm{pH}$ allows for dissociation of the lumenal cargo, which is released into lysosomes by fluid-phase transport, while CI-MPRs recycle to the TGN through retrograde transport. A similar pathway is exploited by surface CI-MPRs following ligand-dependent internalization of M6P-linked cargo [31].

To address the role of IFT20 in intracellular CI-MPR traffic, we carried out a colocalization analysis of CI-MPR with the TGN marker TGN38. A decrease in the TGN localization of CI-MRPs was observed in IFT20KD cells (Fig. 6a) without any change in the plasma membraneassociated pool (Fig. S6a), suggesting a retrograde transport defect. Immunoblot analysis showed an increase in the total CI-MPR in IFT20KD cells (Fig. 6b), which reflects a compensatory cellular response to maintain lysosomal function $[32,33]$. These data rule out a defect in CI-MPR expression in IFT20KD cells and suggest that IFT20 might be implicated in the retrograde transport of CI-MPRs to the TGN. 

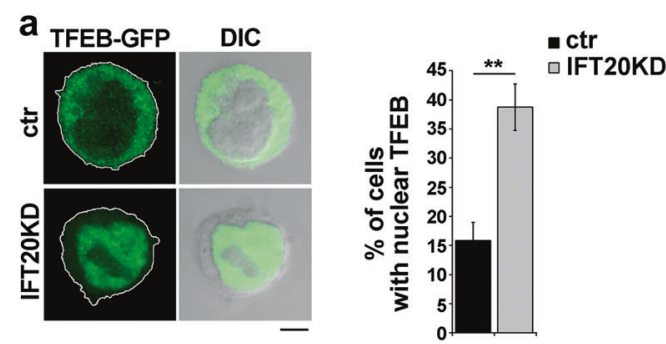

b

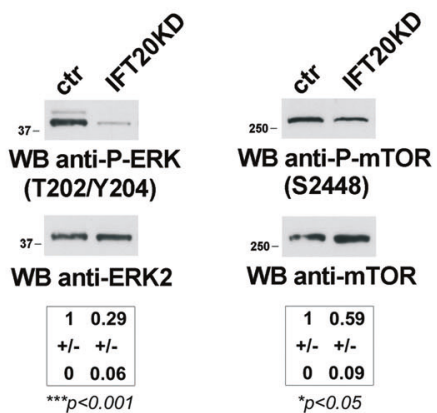

e

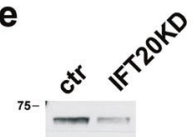

WB anti-P-ZAP-70 (Y319)

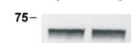

WB anti-ZAP-70

10.26

$+/-+/-$

$\begin{array}{ll}0 & 0.04\end{array}$

$* * * 0<0.001$

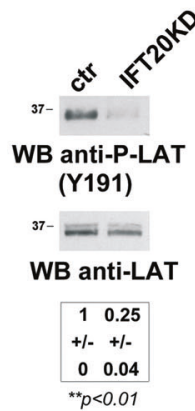

Fig. 4 IFT20 couples tonic TCR signaling to TFEB activation. a Quantification of nuclear GFP-tagged TFEB in ctr and IFT20KD Jurkat cells (mean $\pm \mathrm{SD} ; n=3$; Student's $t$ test). Representative images (medial optical sections) are shown. b Immunoblot analysis with anti-P-ERK1/2 or P-mTOR antibody of lysates from ctr and IFT20KD Jurkat cells. ERK2 or mTOR was used as loading control. The quantification of the relative P-protein levels, normalized to the respective loading controls, is reported below (mean fold $\pm \mathrm{SD}$; ctr value $=1 ; n \geq 3$; one sample $t$ test). c Left, Immunoblot analysis with anti-P-ERK1/2 antibody of lysates from Jurkat cells, treated for $16 \mathrm{~h}$ with $20 \mu \mathrm{M}$ PD098059. ERK2 was used as loading control. Right, Quantitative RT-PCR analysis of TFEB-regulated genes and TFEB in PD098059-treated ctr Jurkat $T$ cells. The relative abundance of gene transcripts was determined on samples using the ddCt method and was normalized to HPRT1. The data (mean $\pm \mathrm{SD} ; n \geq 3$; one sample $t$ test) are expressed as normalized fold expression in treated vs untreated cells (expression in untreated cells set for each gene as 1, dashed line). d Left, Immunoblot analysis with anti-P-mTOR antibody of lysates from Jurkat cells, treated for $16 \mathrm{~h}$ with $250 \mathrm{nM}$ Torin1. mTOR was used as loading control. Right, Quantitative RT-PCR analysis of TFEB-regulated genes and TFEB in Torin1-treated ctr Jurkat T cells. The relative abundance of gene transcripts was determined on samples

To test this hypothesis, we analyzed the retrograde transport of antibody-tagged CI-MRP from the cell surface to the TGN. The rate and extent of ligand-dependent CI-
C
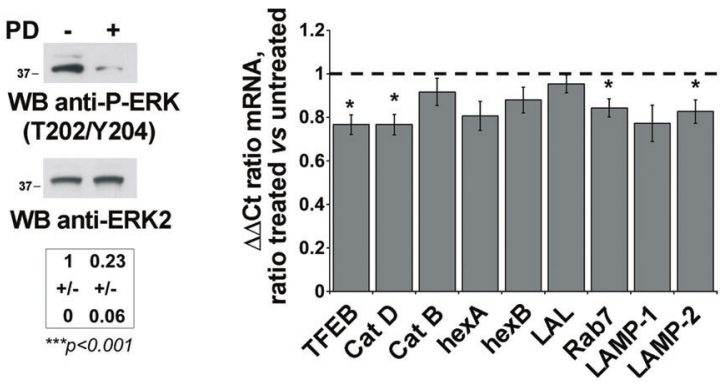

d

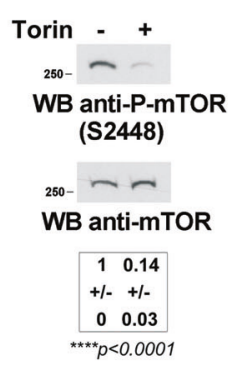

f

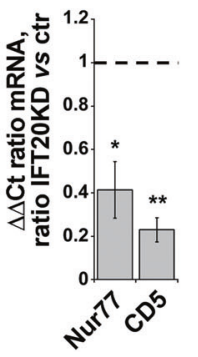

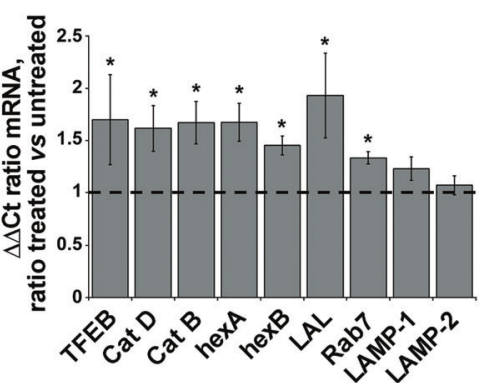

g

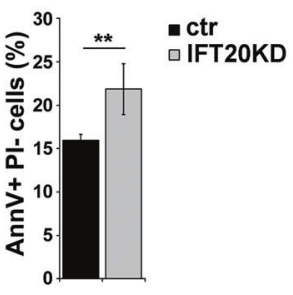

using the ddCt method and was normalized to HPRT1. The data (mean $\pm \mathrm{SD} ; n \geq 3$; one sample $t$ test) are expressed as normalized fold expression in treated vs untreated cells (expression in untreated cells set for each gene as 1, dashed line). e Immunoblot analysis with anti-PZAP-70 (Y319), anti-P-LAT (Y191) or anti-P-Lck (Y505) antibody of lysates from ctr and IFT20KD Jurkat cells. ZAP-70, LAT or Lck was used as loading control. The migration of molecular mass markers is indicated. The quantification of the relative P-protein levels, normalized to the respective loading controls, is reported below (mean fold \pm $\mathrm{SD}$; ctr value $=1 ; n=3$; one sample $t$ test). f Quantitative RT-PCR analysis of Nur77 and CD5 expression in ctr and IFT20KD Jurkat cells. The relative abundance of gene transcripts was determined on triplicate samples using the $\mathrm{ddCt}$ method and was normalized to HPRT1. The data (mean $\pm \mathrm{SD} ; n=3$ ) are expressed as normalized fold expression in IFT20KD vs control cells (expression in ctr cells set for each gene as 1 , dashed line) (one sample $t$ test). g Flow-cytometric analysis of apoptosis in ctr and IFT20KD Jurkat cells grown for $24 \mathrm{~h}$ in the culture medium supplemented with $0.5 \%$ BCS and labeled with Annexin $\mathrm{V}$ and propidium iodide. The histograms show the percentages of Annexin V-positive propidium iodide-negative cells (mean \pm SD; $n=6$; Student's $t$ test). $* P<0.05 ; * * P<0.01 ; * * * P<0.001$; $* * * * P<0.0001$

MRP internalization were found to be comparable between ctr and IFT20KD cells, as assessed by flow-cytometric analysis (Fig. S6b). To track CI-MPRs after their 


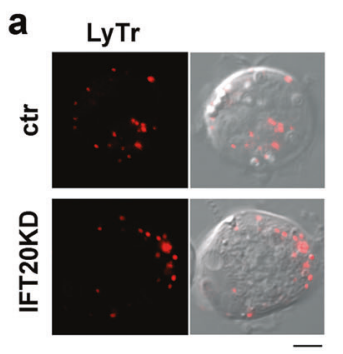

d
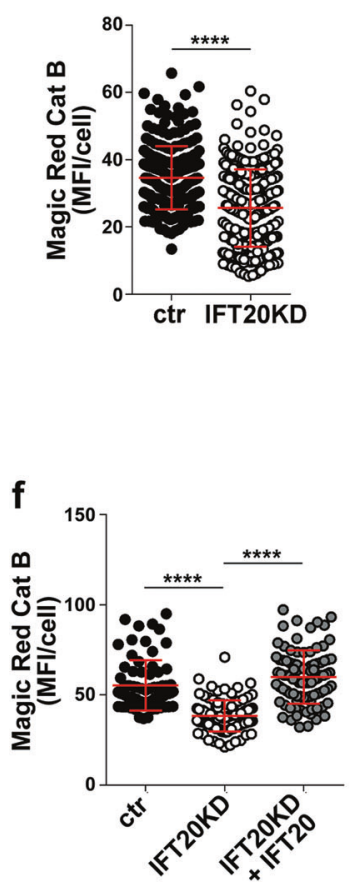

i

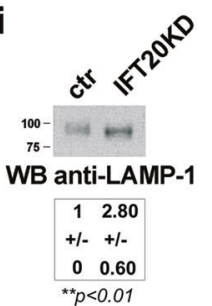

b

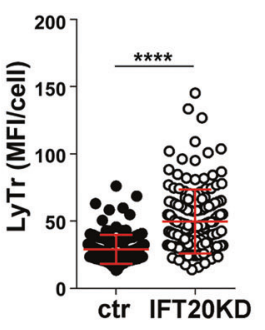

e

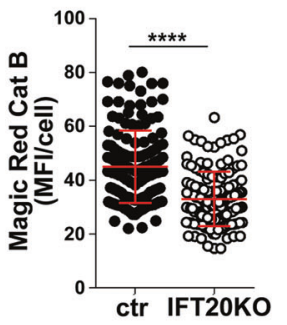

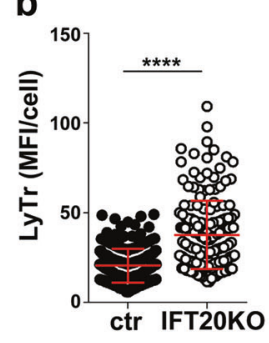

C

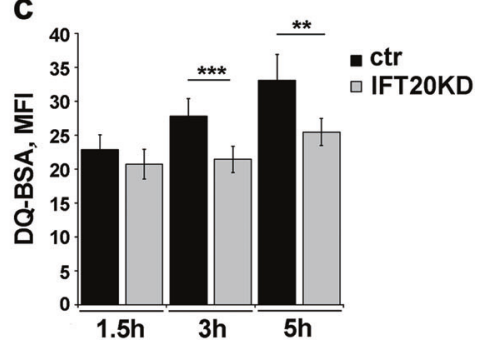

g
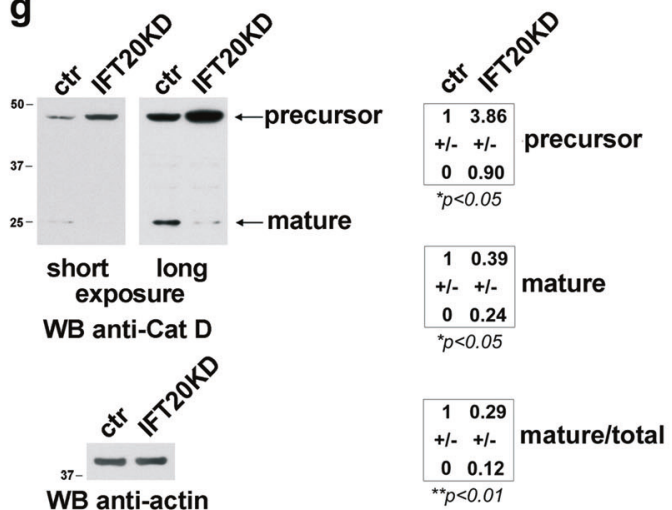

h
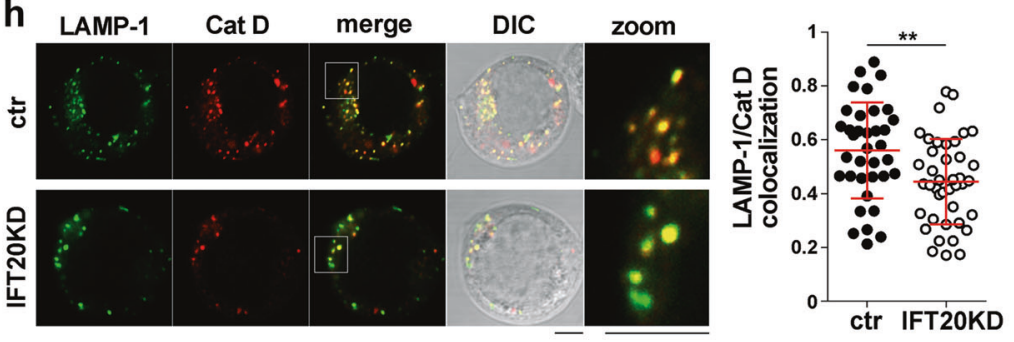

internalization at the plasma membrane, cells were incubated with anti-CI-MPR antibodies at $37^{\circ} \mathrm{C}$ for 30 or $60 \mathrm{~min}$, and CI-MPR-antibody complexes were imaged in permeabilized cells. At the 30 -min time point, endosomes carrying internalized CI-MPRs showed a dispersed, plasma membrane-proximal pattern in both ctr and IFT20KD cells (Fig. S7a). However, at the longer incubation time, CIMRP-positive vesicles remained dispersed in IFT20KD Jurkat cells, at variance with the compact distribution observed in ctr cells (Fig. S7a), as quantified by measuring the distance of CI-MPR-positive vesicles from the centrosome (Fig. 6c). Similar results were obtained on primary
IFT20KO T cells (Fig. 6d; Figs S7b, S3e), indicating a block in retrograde transport in the absence of IFT20. In support of this notion, imaging internalized CI-MPR in cells co-stained for TGN38 showed that at the 60 -min time point, CI-MPRs co-localized with the TGN in ctr cells, but not in IFT20KD cells (Fig. 6e). Of note, the defect in CI-MPR recycling in IFT20KD cells was rescued by forced IFT20 expression (Fig. S7c).

To understand whether the peripherally dispersed vesicles containing internalized CI-MPRs in IFT20KD cells correspond to a specific endosomal compartment, a colocalization analysis was carried out with markers of EEs 
Fig. 5 IFT20 is required for the transport of acid hydrolases to lysosomes. a, b Immunofluorescence analysis of control and IFT20KD Jurkat cells (a) or primary IFT20KO T cells (b) loaded $10 \mathrm{~min}$ at $37^{\circ} \mathrm{C}$ with Lysotracker red (LyTr, $75 \mathrm{nM})$. Median optical sections are shown. Size bar: $5 \mu \mathrm{m}$. The graphs show the quantification of mean fluorescence intensity/cell (MFI/cell) (mean $\pm \mathrm{SD} ; \geq 40$ cells/sample, $n=3$; Mann-Whitney test). c Flow-cytometric analysis of control and IFT20KD Jurkat cells loaded with the probe DQ-BSA $(100 \mu \mathrm{g} / \mathrm{ml})$ for $1.5,3$ or $5 \mathrm{~h}$. The histogram shows the MFI (mean $\pm \mathrm{SD} ; n \geq 4$; Student's $t$ test). d-f Immunofluorescence analysis of control and IFT20KD Jurkat cells ( $\geq 55$ cells/sample, $n=3$; Mann-Whitney test) (d), or primary control and IFT20KO T cells ( $\geq 23$ cells/sample, $n=3$; Kruskal-Wallis test) (e), or IFT20KD Jurkat cells transiently transfected with a construct encoding GFP-tagged IFT20 or control and IFT20KD cells transiently transfected with the respective GFP vector ( $\geq 55$ cells/sample, $n=3$; Mann-Whitney test) (f), loaded $4 \mathrm{~h}$ at $37^{\circ} \mathrm{C}$ with Magic red $(1 \mu \mathrm{M})$. The graphs show the quantification of mean fluorescence intensity/cell (MFI/cell) (mean \pm SD). g Immunoblot analysis of cathepsin D (Santa Cruz antibody) in lysates of ctr and IFT20KD Jurkat cells. Actin was used as loading control. The quantifications of pro-catD (precursor) and mature catD (normalized to actin), and the mature/total catD ratio are shown (mean fold $\pm \mathrm{SD}$; ctr value $=1)(n \geq 3$; one sample $t$ test $)$. h Quantification using Mander's coefficient of the weighted colocalization of LAMP-1 and catD (Abcam antibody) in ctr and IFT20KD Jurkat cells ( $\geq 12$ cells/sample, $n=3$ ). Representative images (medial optical sections) are shown. Size bar: $5 \mu \mathrm{m}$. The data are expressed as mean \pm SD (Student's $t$ test). i Immunoblot analysis of LAMP-1, catD (Abcam antibody), hexosaminidases A (hexA) and B (hexB), and lysosomal acid lipase (LAL) on lysosomes from control and IFT20KD Jurkat cells (equal amounts of proteins from the pool of the fractions enriched in LAMP1 ; see Fig. S1). The quantification of the relative protein expression (mean fold $\pm \mathrm{SD}$; ctr value $=1)$ is reported below $(n=4$; one sample $t$ test). $* P<0.05 ; * * P<0.01 ; * * * P<0.001 ; * * * * P<0.0001$

(Rab5), LEs (Rab7), or recycling endosomes (RE, Rab11). Cells were also stained for Rab9, which participates in MPR recycling from LEs to the TGN [34]. A reduction in the association of recycling CI-MPRs with EEs was observed (Fig. S8a), which was not paralleled by an accumulation in LEs (Fig. S8b), REs (Fig. S8c), or Rab9 ${ }^{+}$endosomes (Fig. S8d). These results suggest that recycling CI-MPRs become trapped in a trafficking-incompetent endosomal compartment.

\section{IFT20 regulates the retrograde traffic of Cl-MPR by recruiting the microtubule motor dynein}

Following the release of lysosomal hydrolases, MPRs are retrieved by the retromer complex and $\mathrm{Rab} 7$ into tubular endosomes that are coupled to microtubules for dyneinmediated retrograde transport [35-39]. One of the main functions of the IFT system in ciliated cells is to promote bidirectional transport of ciliary cargo along the axonemal microtubules by recruiting kinesin and dynein [1], suggesting that this function could be conserved in the nonciliated $\mathrm{T}$ cell.

We investigated the interactions of IFT20 with dynein and CI-MPR in co-immunoprecipitation experiments. IFT20 was found to form a complex with both cytoplasmic dynein (intermediate chain) and CI-MPR, which also included the retromer component VPS35 [40] (Fig. 7a). In addition, IFT20 co-localized with both dynein and CI-MPR, as assessed by confocal imaging (Fig. 7b). The ability of IFT20 to interact with dynein and CI-MPR was confirmed by in vitro GSH-Sepharose pull-down assays using a GSTIFT20 fusion protein (Fig. 7c). The interaction (Fig. 7d) and colocalization (Fig. 7e) of dynein with CI-MPR were impaired in IFT20KD cells, indicating that IFT20 is required for coupling CI-MPR to dynein for retrograde microtubule transport. In support of this notion, treatment of ctr cells with ciliobrevin, a dynein inhibitor, resulted in a peripheral dispersion of recycling CI-MPRs away from the TGN and the centrosome, similar to that observed in IFT20KD cells (Fig. 7f, g). Ciliobrevin treatment of ctr cells also recapitulated the outcome of IFT20 depletion, namely a reduction in the levels of mature catD (Fig. 7h), a basal accumulation of LC3-II and an impaired autophagic flux (Fig. S9). These results support the relevance of the interaction of IFT20 with dynein to its ability to modulate autophagy.

\section{The lysosome-related function of IFT20 is shared by non-ciliated and ciliated cells}

To understand whether the lysosome-related function of IFT20 is specific to $\mathrm{T}$ cells, we extended the analysis to another non-ciliated cell type, the MEC1 B-cell line, as well as to a ciliated cell type, namely the telomeraseimmortalized primary fibroblast line hTERT. Interestingly, similar to IFT20KD T cells, a defect in lysosome biogenesis and function was observed in IFT20KD B cells, including a decrease in the number and an increase in the size of lysosomes (Fig. 8a), impaired catB activity (Fig. 8b), an upregulation in the expression of TFEB-dependent genes (Fig. 8d), an increase in nuclear TFEB (Fig. 8c) and a defect in retrograde CI-MPR trafficking (Fig. 8e). These defects were recapitulated by hTERT fibroblasts transiently knocked out for IFT20 expression by CRISPR/Cas9 gene editing (Fig. 8f-1; Fig. S10a-f). Hence the lysosome-related function of IFT20 is shared by non-ciliated and ciliated cells.

\section{Discussion}

A role for the IFT proteins in vesicular traffic had been initially postulated based on structural similarities with membrane coat components $[41,42]$. IFT20 is indeed implicated in ciliary membrane receptor sorting at the Golgi [11, 43], and also cooperates with BLOC-1 in sorting polycystin-2 from REs for delivery to the cilium [44], underscoring a prominent role for IFT20 in membrane 

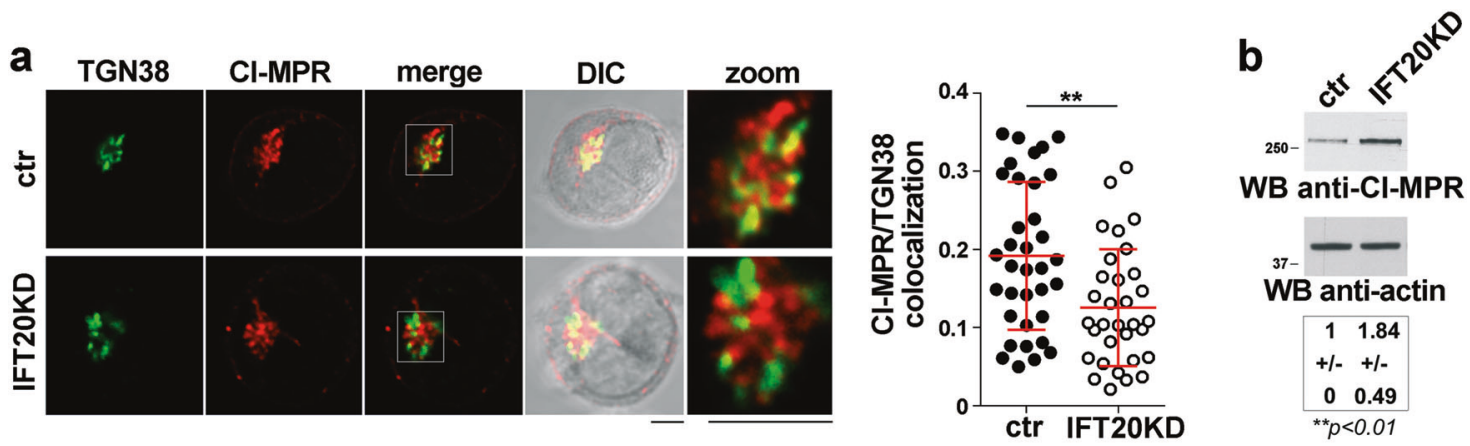

WB anti-CI-MPR
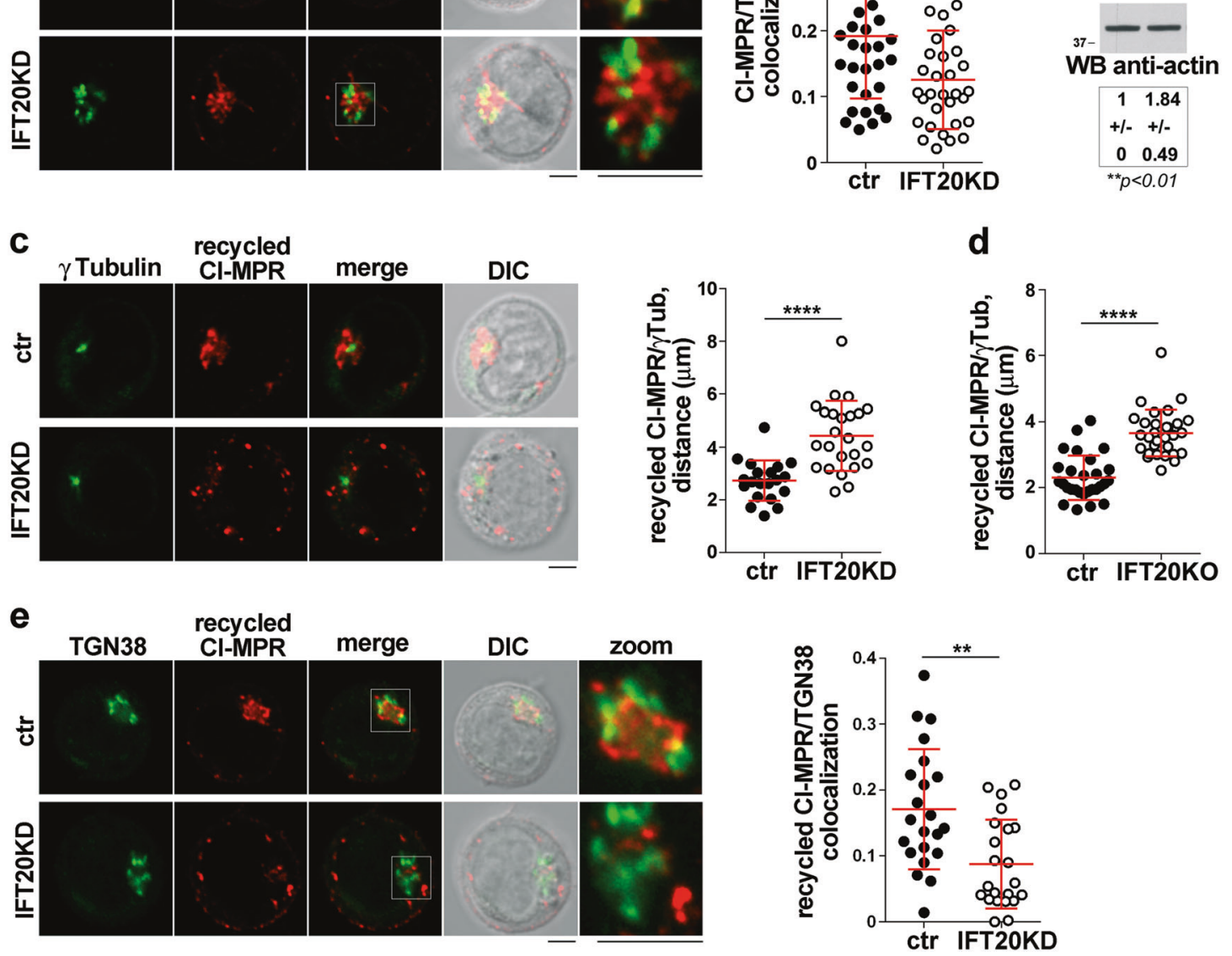

Fig. 6 IFT20 controls the retrograde transport of CI-MPR to the TGN. a Quantification using Mander's coefficient of the weighted colocalization of CI-MPR and the trans-Golgi marker TGN38 in ctr and IFT20KD Jurkat cells ( $\geq 10$ cells/sample, $n \geq 3$ ). Representative images (medial optical sections) are shown. Size bar: $5 \mu \mathrm{m}$. The data are expressed as mean \pm SD (Mann-Whitney test). b Immunoblot analysis with anti-CI-MPR antibody of lysates from ctr and IFT20KD Jurkat cells. Actin was used as loading control. The migration of molecular mass markers is indicated. The quantification of CI-MPR expression, normalized to actin (mean fold $\pm \mathrm{SD}$; ctr value $=1$ ), is reported below ( $n=6$; one sample $t$ test). c, d Immunofluorescence analysis of CIMRP recycling in control and IFT20KD Jurkat cells (c), or primary ctr and IFT20KO T cells (d), incubated with saturating concentrations of receptor-specific $\mathrm{mAb}$ at $37^{\circ} \mathrm{C}$ for $60 \mathrm{~min}$ (Jurkat cells) or $4 \mathrm{~h}$ (primary

trafficking to this organelle. Accumulating evidence supports, however, the notion that the trafficking-related function of the IFT system has been conserved independently of the cell's to build a cilium, as witnessed by our studies in the non-ciliated $\mathrm{T}$ cell $[6-8,45,46]$. Here, we show that IFT20 controls another fundamental process that centrally relies on membrane trafficking, namely lysosome biogenesis and function, by promoting the retrograde trafficking of the CI-MPR on which the lysosomal transport of
$\mathrm{T}$ cells). The histograms show the quantification of the distance between $\mathrm{CI}-\mathrm{MRP}^{+}$vescicles and $\boldsymbol{\gamma}$-tubulin in medial confocal sections (panel c: at least 20 cells from three independent experiments were analyzed, Student's $t$ test; panel $\mathbf{d}$ : at least 28 cells from three independent experiments were analyzed, Mann-Whitney test). e Immunofluorescence analysis of ctr and IFT20KD Jurkat treated as in (c) and stained with anti-TGN38 mAb. The histogram shows the quantification of Mander's colocalization coefficient between CI-MRP ${ }^{+}$ vesicles and TGN38 in medial confocal sections of control or IFT20KD Jurkat cells 60 min after antibody-induced CI-MPR internalization. At least 22 cells from three independent experiments were analyzed (Mann-Whitney test). Representative images are shown. Size bar: $5 \mu \mathrm{m} . * * P<0.01 ; * * * * P<0.0001$

acid hydrolases centrally depends, and provide evidence that this function is achieved through coupling CI-MPRs to the microtubule motor dynein (schematized in Fig. S11).

While the IFT system has been implicated in the ciliary localization of the autophagic machinery in ciliated cells [10], our findings highlight a novel function for IFT20 in $\mathrm{T}$ cells that is relevant to lysosome functionality and hence impacts on the last step of autophagy, namely the clearance of autophagolysosome cargo, including intraluminal LC3-II 
a

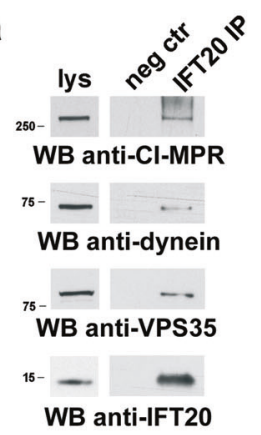

C

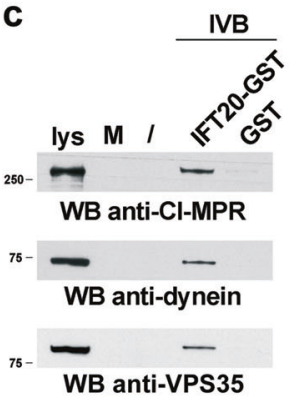

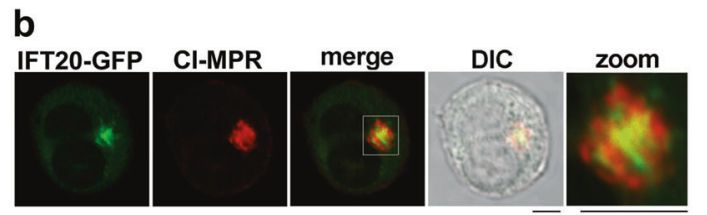

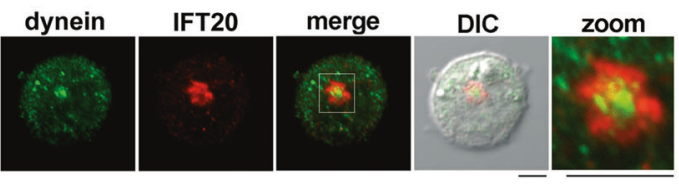

d neg ctr $\stackrel{\substack{\text { dynein } \\ \mathrm{IP}}}{\mathrm{s}}$

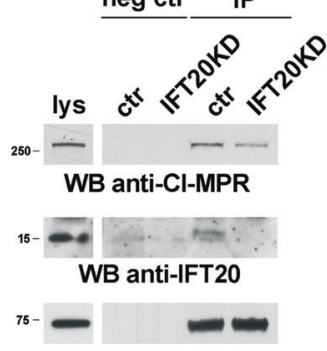

WB anti-dynein
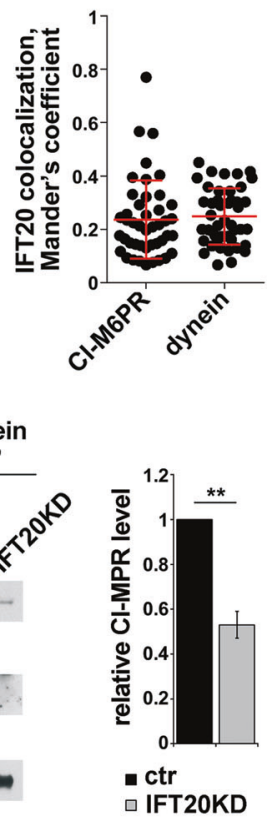
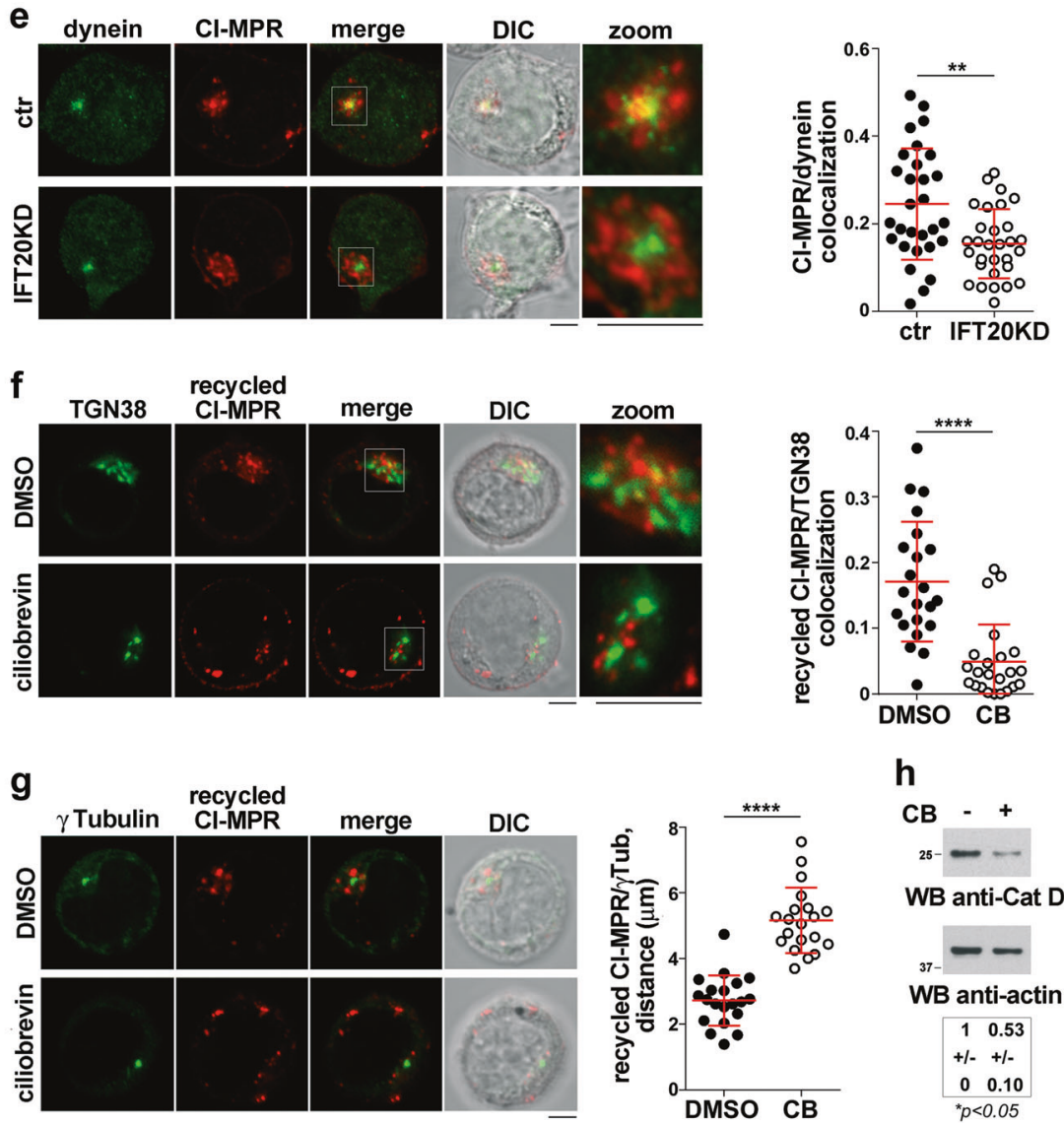

h

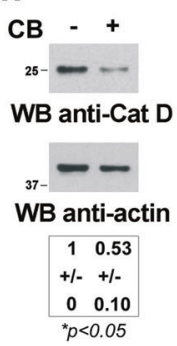

and LDs. The lysosomal defect in IFT20-deficient T cells is supported by the increase in lysosome size, resulting from undegraded material accumulation, the LD accumulation, and the compensatory attempt to correct the defect involving activation of the TFEB-driven lysosome biogenesis program [24]. The fact that lysosomes remain punctate in 
Fig. 7 IFT20 couples CI-MPR to the microtubule motor dynein. a Immunoblot analysis using anti-CI-MPR, anti-dynein or anti-VPS35 antibodies of IFT20-specific immunoprecipitates from lysates of Jurkat cells. A preclearing control was included in each blot (neg ctr). A total cell lysate was included in each gel to identify the migration of the tested proteins. The immunoblot shown is representative of three independent experiments. A shorter exposure of the total lysate is shown. b Quantification of Mander's colocalization coefficient (mean $\pm \mathrm{SD} ; 44$ cells, $n \geq 3$ ) between dynein (right) or CI-MPR (left) with IFT20 in medial confocal sections of Jurkat cells stained with the respective antibodies (for dynein colocalization) or Jurkat cells transiently transfected with the IFT20-GFP construct and stained with antiCI-MPR antibodies. Representative images are shown. Size bar: $5 \mu \mathrm{m}$. c Immunoblot analysis with anti-CI-MPR, anti-dynein or anti-VPS35 antibodies of in vitro-binding assays carried out on post-nuclear supernatants of Jurkat cells using IFT20-GST fusion protein. The Ponceau red staining of the same filter is shown to compare the levels of GST-IFT20 and GST used in the assay. The immunoblot shown is representative of three independent experiments. d Immunoblot analysis with anti-CI-MPR and anti-IFT20 antibodies of dynein-specific immunoprecipitates from lysates of ctr and IFT20KD Jurkat cells. A preclearing control was included in each blot (neg ctr). A total cell lysate was included in each gel to identify the migration of the tested proteins. A shorter exposure of the total lysate is shown. The histogram shows the quantification of CI-MPR normalized to the immunoprecipitated dynein (mean fold $\pm \mathrm{SD}$; control value $=1, n=3$; one sample $t$ test). e Quantification of Mander's colocalization coefficient (mean $\pm \mathrm{SD} ; \geq 29$ cells, $n=3$; Student's $t$ test) between dynein and CIMPR in medial confocal sections of control or IFT20KD Jurkat cells stained with the respective antibodies. Representative images are shown. Size bar: $5 \mu \mathrm{m}$. f, $\mathbf{g}$ Quantification of Mander's colocalization coefficient between CI-MRP ${ }^{+}$vesicles and TGN38 (f) $(\geq 22$ cells, $n=$ 3; Mann-Whitney test) and of the distance between CI-MRP ${ }^{+}$vesicles and $\gamma$-tubulin (g) (20 cells, $n=3$; Student's $t$ test) in medial confocal sections of Jurkat cells incubated with saturating concentrations of CIMRP $\mathrm{mAb}$ at $37^{\circ} \mathrm{C}$ for $60 \mathrm{~min}$ in the presence or absence of the dynein inhibitor ciliobrevin $(\mathrm{CB}, 50 \mu \mathrm{M})$. The data are expressed as mean \pm SD. Size bar: $5 \mu \mathrm{m}$. h Immunoblot analysis of catD (Abcam antibody) in lysates of ctr and IFT20KD Jurkat cells untreated or treated with the ciliobrevin $(\mathrm{CB}, 50 \mu \mathrm{M})$ for $16 \mathrm{~h}$. Actin was used as loading control. The quantification of mature catD is reported below (mean fold $\pm \mathrm{SD}$; ctr value $=1)(n=3$; one sample $t$ test $) . * P<0.05$; $* * P<0.01 ; * * * * P<0.0001$

IFT20KD cells rules out reduced lysosome tubulation/ reformation as the cause of their observed enlargement.

The nuclear localization of TFEB is negatively controlled by ERK and mTOR [25, 26], both of which have a lower basal activity in IFT20KD T cells. The fact that inhibition of mTOR, but not of ERK, recapitulates the effects of IFT20 deficiency on TFEB-dependent gene transcription selectively implicates mTOR in TFEB activation in $\mathrm{T}$ cells. Interestingly, while lysosomal dysfunction appears sufficient to cause TFEB translocation to the nucleus [23], the decrease in mTOR and ERK activity in IFT20KD cells is associated with a decreased activation status of upstream components in the TCR signaling cascade, as well as expression of the tonic signaling markers CD5 and Nur77, indicating that TCR dysfunction may underlie the enhanced nuclear TFEB translocation and highlighting an unexpected connection between basal TCR signaling and lysosome biogenesis.
TCR signaling can lead to different biological outcomes, ranging from activation and differentiation, to activation-induced cell death, to survival, depending on a combination of factors that include ligand affinity, signal duration and co-engagement of co-stimulatory or coinhibitory receptors [47]. We have reported that IFT20 is required for TCR signaling and immune synapse assembly $[6,7]$, a function that impacts on in vivo peripheral T-cell activation and differentiation [8]. Although no gross alterations in peripheral $\mathrm{T}$-cell subsets were detected in mice with a $\mathrm{T}$ cell-specific knockout [8], the fact that IFT20 deficiency impacts on tonic signaling and homeostatic T-cell survival in vitro warrants an in-depth analysis of these features in the complex context of in vivo T-cell homeostasis.

The basic pathway of retrograde transport of CI-MPRs following the release of acid hydrolases in LEs has been in part characterized. Recycling CI-MPRs are retrieved into tubular endosomes by the retromer complex [35, 36, 48] and traffic to the TGN with the assistance of several Rab GTPases and their effectors, and of the STX16/Vti1A/ VAMP-3 SNARE complex [49, 50]. Our results identify IFT20 as a new component of the CI-MPR retrograde pathway, as supported by the decrease in CI-MPR colocalization with the TGN under basal conditions and the failure of antibody-tagged recycling CI-MPRs to reach the TGN. A similar phenotype, with dispersion of recycling CI-MPRs away from the TGN, has been reported in cells deficient for components of the retromer complex and other regulators of retrograde traffic [32, 33, 51-56].

The transport of retromer-associated membrane cargoes from endosomes to the TGN requires their association with the dynein/dynactin minus-end microtubule motor complex. This is achieved through the interaction of the retromer components SNX5/SNX6 with dynactin [37, 38], with a switch along the pathway to other dynein/dynactin adaptors, such as the SNARE Snapin [57]. We found that the CI-MPR retrograde transport defect in IFT20-deficient $\mathrm{T}$ cells is associated with an impairment in the ability of CI-MPR to interact with dynein. Taken together with the finding that IFT20 forms a complex with both dynein and CI-MPR, as well as the retromer component VPS35, our results highlight IFT20 as a new dynein adaptor that allows recycling CI-MPRs to move along the microtubules during retrograde transport. We previously identified ARPC 3, a component of the Arp2/3 actin adaptor complex that is required for retrograde transport [51], as a molecular partner of IFT20 [58]. Since the cytosolic tail of the CI-MPR does not contain sequences known to be recognized by IFT20 [59], IFT20 may be recruited to recycling CI-MPRs by Arp2/3, coupling in turn these endosomes to microtubules by binding to dynein downstream of SNX5/SNX6. 
a

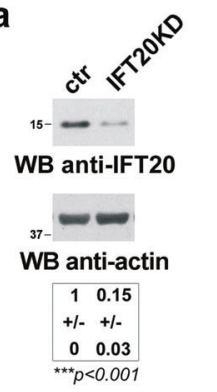

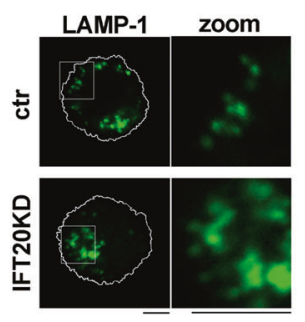

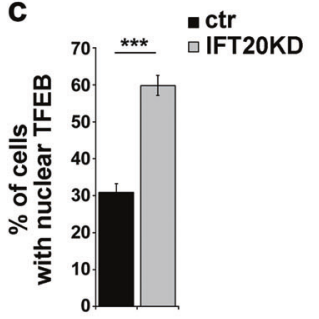

d

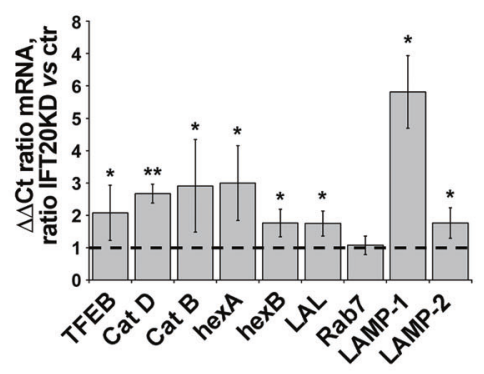

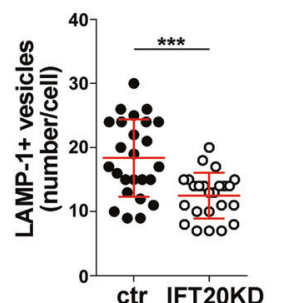

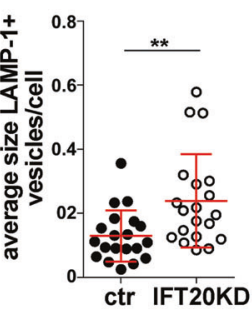

b

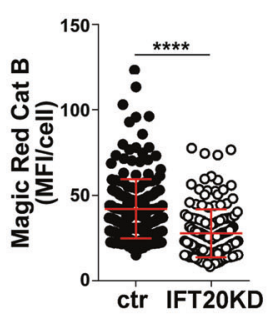

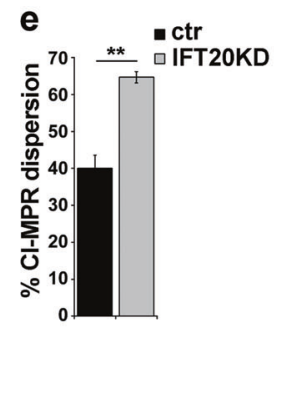
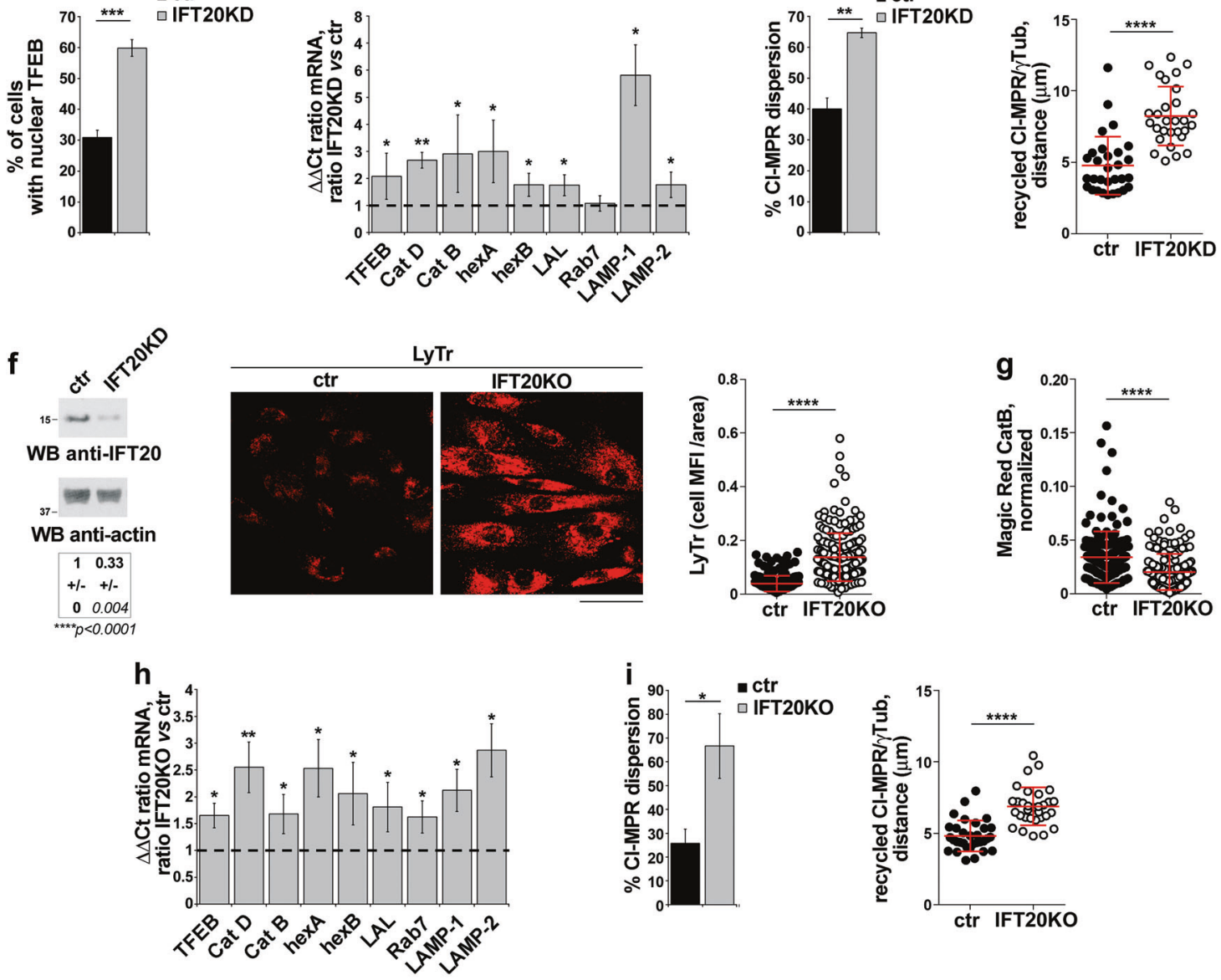

I
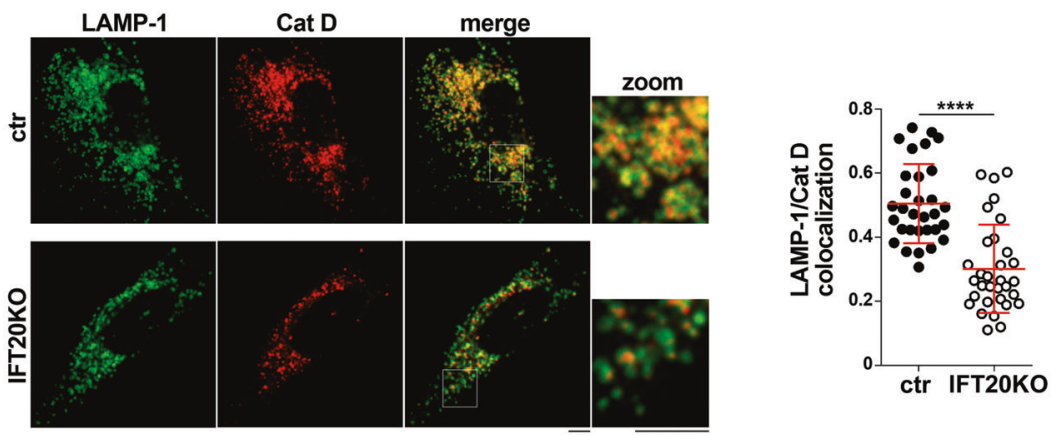
Fig. 8 IFT20 controls lysosome biogenesis in non-ciliated and ciliated cells. a Representative anti-IFT20 immunoblot of lysates of ctr and IFT20KD MEC1 B cells. Actin was used as loading control. The migration of molecular mass markers is indicated. The quantification of the relative protein expression, normalized to actin (mean fold $\pm \mathrm{SD}$; ctr value $=1)$, is reported below $(n=3$; one sample t test). Right, Immunofluorescence analysis of LAMP-1 in ctr and IFT20KD MEC1 $B$ cells. The graphs show the quantification of the number and average size $\left(\mu \mathrm{m}^{2}\right)$ of LAMP- $1^{+}$vesicles/cell (mean $\pm \mathrm{SD}$, Mann-Whitney test). At least 20 cells from three independent experiments were analyzed. b Immunofluorescence analysis of control and IFT20KD MEC1 cells loaded $4 \mathrm{~h}$ at $37^{\circ} \mathrm{C}$ with Magic red $(1 \mu \mathrm{M})$. The histograms show the quantification of mean fluorescence intensity/cell (MFI/cell) (mean $\pm \mathrm{SD} ; \geq 50$ cells/sample, $n=3$; Mann-Withney test). c Quantification of nuclear GFP-tagged TFEB in ctr and IFT20KD MEC 1 cells (mean $\pm \mathrm{SD} ; n=3$; Student's $t$ test). d Quantitative RTPCR analysis of TFEB-regulated genes and TFEB in control and IFT20KD MEC1 cells. The relative abundance of gene transcripts was determined on triplicate samples using the ddCt method and was normalized to HPRT1. The data (mean $\pm \mathrm{SD} ; n \geq 3$; one sample $t$ test) are expressed as normalized fold expression in IFT20KD vs control vs cells (expression in ctr cells set for each gene as 1, dashed line). e Immunofluorescence analysis of CI-MRP recycling in control and IFT20KD MEC1 cells, incubated with saturating concentrations of receptor-specific $\mathrm{mAb}$ at $37{ }^{\circ} \mathrm{C}$ for $60 \mathrm{~min}$ and stained with anti- $\gamma$ tubulin antibodies. Left, Quantification of the percentage of CI-MRP dispersion in control and IFT20KD MEC cells, calculated as percentage of cells where more than $40 \%$ of CI-MRP ${ }^{+}$vesicles are distant more than $2.5 \mu \mathrm{m}$ from the centrosome in individual medial confocal sections (mean $\pm \mathrm{SD}, n=3$; Student's $t$ test). Right, Quantification of the distance between CI-MRP ${ }^{+}$vescicles and $\gamma$-tubulin in medial confocal sections of control or IFT20KD MEC1 cells (mean \pm SD; $n$ $=3$; Mann-Withney test). f Representative anti-IFT20 immunoblot of lysates of ctr and IFT20KD hTERT fibroblasts transiently knocked out for IFT20 expression by CRISPR/Cas9 gene editing. Actin was used as loading control. The migration of molecular mass markers is

It is noteworthy that the lysosome-related function of IFT20, including the regulation of CI-MPR recycling and the ability to modulate the TFEB-dependent lysosome biogenesis program, is not limited to $\mathrm{T}$ cells but extends to other non-ciliated as well ciliated cells. Hence, our results implicate the ciliogenesis protein IFT20 as a central player in a fundamental, cilium-independent cellular function, and underscore the trafficking-related role of the IFT system beyond ciliogenesis. In addition, they contribute to unravel the general and cell-specific mechanisms that coordinate lysosome biogenesis and function.

Acknowledgements The authors wish to thank Greg Pazour for critically reading the paper and for generously providing anti-IFT20 antibodies and constructs, Roman Polishchuk for critically reading the paper and for his help on the electron microscopy analysis. They also wish to thank Francesco Cecconi, Claire Hivroz, Laurence Bataille, Roberto Zoncu, Diego Medina, Alberto Luini, Francesco Nardi, and Marco Telford for useful discussions and suggestions. The GFP-TFEB construct was generously provided by Diego Medina and the hTERT cells by Paola Marcolongo. This work was carried out with the generous support of Telethon - Italy (Grant GGP16003). The CTB lab is also supported by a grant from AIRC (Grant IG 20148). VC is supported by the Lundbeck Foundation indicated. The quantification of the relative protein expression, normalized to actin (mean fold $\pm \mathrm{SD}$; ctr value $=1$ ), is reported below $(n=3$; one sample $t$ test). Right, Immunofluorescence analysis of control and IFT20KO hTERT fibroblasts loaded $10 \mathrm{~min}$ at $37^{\circ} \mathrm{C}$ with Lysotracker red (LyTr, $75 \mathrm{nM}$ ). Median optical sections are shown. Size bar: $50 \mu \mathrm{m}$. The graphs show the quantification of the mean fluorescence intensity (MFI)/cell normalized to the cell area (mean \pm $\mathrm{SD} ; \geq 35$ cells/sample, $\mathrm{n}=5$; Mann-Whitney test). g Immunofluorescence analysis of control and IFT20KO hTERT fibroblasts loaded $4 \mathrm{~h}$ at $37^{\circ} \mathrm{C}$ with Magic Red $(1 \mu \mathrm{M})$. The graphs show the quantification of cell MFI/area normalized to the Lysotracker MFI (right) in ctr and IFT20KO cells (mean $\pm \mathrm{SD}$; $\geq 29$ cells/sample, $n=5$; Mann-Whitney test). h Quantitative RT-PCR analysis of TFEBregulated genes and TFEB in control and IFT20KO hTERT fibroblasts gene-edited by CRISPR-Cas 9 technology $(n \geq 3$; one sample $\mathrm{t}$ test). The relative abundance of gene transcripts was determined on triplicate samples using the ddCt method and was normalized to HPRT1. The data (mean $\pm \mathrm{SD}$ ) are expressed as normalized fold expression in IFT20KO vs control cells (expression in ctr cells set for each gene as 1 , dashed line). i Immunofluorescence analysis of CI-MRP recycling in control and IFT20KO hTERT fibroblasts, incubated with saturating concentrations of receptor-specific $\mathrm{mAb}$ at $37^{\circ} \mathrm{C}$ for $4 \mathrm{~h}$ and stained with anti- $\gamma$-tubulin antibodies. Left, the data are quantified as the percentage of CI-MRP dispersion (mean $\pm \mathrm{SD}$ ), calculated as percentage of cells where at least $40 \%$ of CI-MRP ${ }^{+}$vesicles are distant more than $2.5 \mu \mathrm{m}$ from the centrosome in individual medial confocal sections (mean $\pm \mathrm{SD} ; n=3$; Student's $t$ test). Right, quantification of the distance between CI-MRP ${ }^{+}$vescicles and $\gamma$-tubulin in medial confocal sections of control or IFT20KO hTERT fibroblasts (mean \pm $\mathrm{SD}$; $\geq 31$ cells, $n=3$; Mann-Whitney test). I Quantification using Mander's coefficient of the weighted colocalization of LAMP-1 and catD (Abcam antibody) in ctr and IFT20KO hTERT fibroblasts ( $\geq 10$ cells/sample, $n=3$ ). Representative images (medial optical sections) are shown. Size bar: $5 \mu \mathrm{m}$. The data are expressed as mean \pm SD (Mann-Whitney test). $* P<0.05 ; \quad * * P<0.01 ; \quad * * * P<0.001$; $* * * * P<0.0001$

(R209-2015-3505) and the KBVU from the Danish Cancer Society (R146-A9471).

\section{Compliance with ethical standards}

Conflict of interest The authors declare that they have no conflict of interest.

Publisher's note: Springer Nature remains neutral with regard to jurisdictional claims in published maps and institutional affiliations.

\section{References}

1. Pedersen LB, Rosenbaum JL. Intraflagellar transport (IFT) role in ciliary assembly, resorption and signalling. Curr Top Dev Biol. 2008;85:23-61.

2. Prevo B, Scholey JM, Peterman EJG. Intraflagellar transport: mechanisms of motor action, cooperation, and cargo delivery. FEBS J. 2017;284:2905-31.

3. Mourao A, Christensen ST, Lorentzen E. The intraflagellar transport machinery in ciliary signaling. Curr Opin Struct Biol. 2016;41:98-108.

4. Soares H, Lasserre R, Alcover A. Orchestrating cytoskeleton and intracellular vesicle traffic to build functional immunological synapses. Immunol Rev. 2013;256:118-32. 
5. Dustin ML, Choudhuri K. Signaling and polarized communication across the $\mathrm{T}$ cell immunological synapse. Annu Rev Cell Dev Biol. 2016;32:303-25.

6. Finetti F, Paccani SR, Riparbelli MG, Giacomello E, Perinetti G, Pazour GJ, et al. Intraflagellar transport is required for polarized recycling of the TCR/CD3 complex to the immune synapse. Nat Cell Biol. 2009;11:1332-9.

7. Finetti F, Patrussi L, Masi G, Onnis A, Galgano D, Lucherini OM, et al. Specific recycling receptors are targeted to the immune synapse by the intraflagellar transport system. J Cell Sci. 2014;127 (Pt 9):1924-37.

8. Vivar OI, Masi G, Carpier JM, Magalhaes JG, Galgano D, Pazour GJ et al. IFT20 controls LAT recruitment to the immune synapse and T-cell activation in vivo. Proc Natl Acad Sci USA. 2016; 113:386-91.

9. Satir P, Mitchell DR, Jekely G. How did the cilium evolve? Curr Top Dev Biol. 2008;85:63-82.

10. Pampliega O, Orhon I, Patel B, Sridhar S, Díaz-Carretero A, Beau I, et al. Functional interaction between autophagy and ciliogenesis. Nature. 2013;502:194-200.

11. Follit JA, Tuft RA, Fogarty KE, Pazour GJ. The intraflagellar transport protein IFT20 is associated with the Golgi complex and is required for cilia assembly. Mol Biol Cell. 2006;17:3781-92.

12. Stacchini A, Aragno M, Vallario A, Alfarano A, Circosta P, Gottardi D, et al. MEC1 and MEC2: two new cell lines derived from B-chronic lymphocytic leukaemia in prolymphocytoid transformation. Leuk Res. 1999;23:127-36.

13. Kimura S, Noda T, Yoshimori T. Dissection of the autophagosome maturation process by a novel reporter protein, tandem fluorescent-tagged LC3. Autophagy. 2007;3:452-60.

14. Ran FA, Hsu PD, Wright J, Agarwala V, Scott DA, Zhang F. Genome engineering using the CRISPR-Cas9 system. Nat Protoc. 2013;8:2281-308.

15. Pazour GJ, Baker SA, Deane JA, Cole DG, Dickert BL, Rosenbaum JL, et al. The intraflagellar transport protein, IFT88, is essential for vertebrate photoreceptor assembly and maintenance. J Cell Biol. 2002;157:103-13.

16. Bolte S, Cordelieres FP. A guided tour into subcellular colocalization analysis in light microscopy. J Microsc. 2006;224(Pt 3): 213-32.

17. Abdollahzadeh I, Schwarten M, Gensch T, Willbold D, Weiergraber $\mathrm{OH}$. The Atg8 family of proteins-modulating shape and functionality of autophagic membranes. Front Genet. 2017;8:109.

18. Yu L, Chen Y, Tooze SA. Autophagy pathway: cellular and molecular mechanisms. Autophagy. 2018:14:207-215.

19. Platt FM, Boland B, van der Spoel AC. The cell biology of disease: lysosomal storage disorders: the cellular impact of lysosomal dysfunction. J Cell Biol. 2012;199:723-34.

20. Martin S, Parton RG. Lipid droplets: a unified view of a dynamic organelle. Nat Rev Mol Cell Biol. 2006;7:373-8.

21. Singh R, Kaushik S, Wang Y, Xiang Y, Novak I, Komatsu M, et al. Autophagy regulates lipid metabolism. Nature. 2009;458: 1131-5.

22. Settembre C, Fraldi A, Medina DL, Ballabio A. Signals from the lysosome: a control centre for cellular clearance and energy metabolism. Nat Rev Mol Cell Biol. 2013;14:283-96.

23. Sardiello M, Palmieri M, di Ronza A, Medina DL, Valenza M, Gennarino VA, et al. A gene network regulating lysosomal biogenesis and function. Science. 2009;325:473-7.

24. Palmieri M, Impey S, Kang H, di Ronza A, Pelz C, Sardiello M, et al. Characterization of the CLEAR network reveals an integrated control of cellular clearance pathways. Hum Mol Genet. 2011;20:3852-66.

25. Settembre C, Di Malta C, Polito VA, Garcia Arencibia M, Vetrini F, Erdin S, et al. TFEB links autophagy to lysosomal biogenesis. Science. 2011;332:1429-33.
26. Settembre C, Zoncu R, Medina DL, Vetrini F, Erdin S, Erdin S, et al. A lysosome-to-nucleus signalling mechanism senses and regulates the lysosome via mTOR and TFEB. EMBO J. 2012;31: 1095-108.

27. Myers DR, Zikherman J, Roose JP. Tonic signals: why do lymphocytes bother? Trends Immunol. 2017;38:844-57.

28. Shacka JJ, Roth KA. Cathepsin D deficiency and NCL/Batten disease: there's more to death than apoptosis. Autophagy. 2007;3: 474-6.

29. Siklos M, BenAissa M, Thatcher GR. Cysteine proteases as therapeutic targets: does selectivity matter? A systematic review of calpain and cathepsin inhibitors. Acta Pharm Sin B. 2015;5: 506-19.

30. Zaidi N, Maurer A, Nieke S, Kalbacher H. Cathepsin D: a cellular roadmap. Biochem Biophys Res Commun. 2008;376:5-9.

31. Braulke T, Bonifacino JS. Sorting of lysosomal proteins. Biochim Biophys Acta. 2009;1793:605-14.

32. Ganley IG, Carroll K, Bittova L, Pfeffer S. Rab9 GTPase regulates late endosome size and requires effector interaction for its stability. Mol Biol Cell. 2004;15:5420-30.

33. Progida C, Cogli L, Piro F, De Luca A, Bakke O, Bucci C. Rab7b controls trafficking from endosomes to the TGN. J Cell Sci. 2010;123(Pt 9):1480-91.

34. Riederer MA, Soldati T, Shapiro AD, Lin J, Pfeffer SR. Lysosome biogenesis requires Rab9 function and receptor recycling from endosomes to the trans-Golgi network. J Cell Biol. 1994;125: 573-82.

35. Seaman MN. Cargo-selective endosomal sorting for retrieval to the Golgi requires retromer. J Cell Biol. 2004;165:111-22.

36. Arighi CN, Hartnell LM, Aguilar RC, Haft CR, Bonifacino JS. Role of the mammalian retromer in sorting of the cationindependent mannose 6-phosphate receptor. J Cell Biol. 2004; $165: 123-33$.

37. Rojas R, van Vlijmen T, Mardones GA, Prabhu Y, Rojas AL, Mohammed S, et al. Regulation of retromer recruitment to endosomes by sequential action of Rab5 and Rab7. J Cell Biol. 2008;183:513-26.

38. Wassmer T, Attar N, Harterink M, van Weering JR, Traer CJ, Oakley J, et al. The retromer coat complex coordinates endosomal sorting and dynein-mediated transport, with carrier recognition by the trans-Golgi network. Dev Cell. 2009;17:110-22.

39. Hong Z, Yang Y, Zhang C, Niu Y, Li K, Zhao X, et al. The retromer component SNX6 interacts with dynactinp150(Glued) and mediates endosome-to-TGN transport. Cell Res. 2009;19: 1334-49.

40. Burd C, Cullen PJ. Retromer: a master conductor of endosome sorting. Cold Spring Harb Perspect Biol. 2014;6:a016774.

41. Avidor-Reiss T, Maer AM, Koundakjian E, Polyanovsky A, Keil $\mathrm{T}$, Subramaniam S, et al. Decoding cilia function: defining specialized genes required for compartmentalized cilia biogenesis. Cell. 2004;117:527-39.

42. Jekely G, Arendt D. Evolution of intraflagellar transport from coated vesicles and autogenous origin of the eukaryotic cilium. Bioessays . 2006;28:191-8.

43. Keady BT, Le YZ, Pazour GJ. IFT20 is required for opsin trafficking and photoreceptor outer segment development. Mol Biol Cell. 2011;22:921-30.

44. Monis WJ, Faundez V, Pazour GJ. BLOC-1 is required for selective membrane protein trafficking from endosomes to primary cilia. J Cell Biol. 2017;216:2131-50.

45. Onnis A, Finetti F, Patrussi L, Gottardo M, Cassioli C, Spanò S, et al. The small GTPase Rab29 is a common regulator of immune synapse assembly and ciliogenesis. Cell Death Differ. 2015;22: 1687-99.

46. Finetti F, Patrussi L, Galgano D, Cassioli C, Perinetti G, Pazour GJ, et al. The small GTPase Rab8 interacts with VAMP-3 to 
regulate the delivery of recycling $\mathrm{T}$-cell receptors to the immune synapse. J Cell Sci. 2015;128:2541-52.

47. Hogquist KA, Jameson SC. The self-obsession of T cells: how TCR signaling thresholds affect fate 'decisions' and effector function. Nat Immunol. 2014;15:815-23.

48. Kvainickas A, Jimenez-Orgaz A, Nagele $\mathrm{H}, \mathrm{Hu} \mathrm{Z}$, Dengjel J, Steinberg F. Cargo-selective SNX-BAR proteins mediate retromer trimer independent retrograde transport. J Cell Biol. 2017;216: 3677-93.

49. Lu L, Hong W. From endosomes to the trans-Golgi network. Semin Cell Dev Biol. 2014;31:30-9.

50. Pfeffer SR. Entry at the trans-face of the Golgi. Cold Spring Harb Perspect Biol. 2011;3:1-11.

51. Gomez TS, Billadeau DD. A FAM21-containing WASH complex regulates retromer-dependent sorting. Dev Cell. 2009;17:699-711.

52. Kirkbride KC, Hong NH, French CL, Clark ES, Jerome WG, Weaver AM. Regulation of late endosomal/lysosomal maturation and trafficking by cortactin affects Golgi morphology. Cytoskelet (Hoboken). 2012;69:625-43.

53. Espinosa EJ, Calero M, Sridevi K, Pfeffer SR. RhoBTB3: a Rho GTPase-family ATPase required for endosome to Golgi transport. Cell. 2009;137:938-48.
54. Meyer C, Zizioli D, Lausmann S, Eskelinen EL, Hamann J, Saftig P, et al. mu1A-adaptin-deficient mice: lethality, loss of AP-1 binding and rerouting of mannose 6-phosphate receptors. EMBO J. 2000;19:2193-203.

55. Lieu ZZ, Derby MC, Teasdale RD, Hart C, Gunn P, Gleeson PA. The golgin GCC88 is required for efficient retrograde transport of cargo from the early endosomes to the trans-Golgi network. Mol Biol Cell. 2007;18:4979-91.

56. Perez-Victoria FJ, Mardones GA, Bonifacino JS. Requirement of the human GARP complex for mannose 6-phosphate-receptordependent sorting of cathepsin D to lysosomes. Mol Biol Cell. 2008;19:2350-62.

57. Cai Q, Lu L, Tian JH, Zhu YB, Qiao H, Sheng ZH. Snapinregulated late endosomal transport is critical for efficient autophagy-lysosomal function in neurons. Neuron. 2010;68: $73-86$.

58. Galgano D, Onnis A, Pappalardo E, Galvagni F, Acuto O, Baldari CT. The $\mathrm{T}$ cell IFT20 interactome reveals new players in immune synapse assembly. J Cell Sci. 2017;130: 1110-21.

59. Pazour GJ, Bloodgood RA. Targeting proteins to the ciliary membrane. Curr Top Dev Biol. 2008;85:115-49. 